\title{
Molecular abundances in carbon-rich circumstellar envelopes ${ }^{\star}$
}

\author{
P. M. Woods ${ }^{1,2}$, F. L. Schöier ${ }^{3}$, L.-Å. Nyman ${ }^{1,4}$, and H. Olofsson ${ }^{5}$ \\ 1 European Southern Observatory, Alonso de Cordova 3107, Casilla 19001, Santiago 19, Chile \\ 2 Department of Physics, UMIST, PO Box 88, Manchester M60 1QD, UK \\ ${ }^{3}$ Leiden Observatory, PO Box 9513, 2300 RA Leiden, The Netherlands \\ 4 Onsala Space Observatory, 43992 Onsala, Sweden \\ 5 Stockholm Observatory, AlbaNova, 10691 Stockholm, Sweden
}

Received 25 September 2002 / Accepted 4 February 2003

\begin{abstract}
A millimetre molecular line survey of seven high mass-loss rate carbon stars in both the northern and southern skies is presented. A total of 196 emission lines (47 transitions) from 24 molecular species were detected. The observed CO emission is used to determine mass-loss rates and the physical structure of the circumstellar envelope, such as the density and temperature structure, using a detailed radiative transfer analysis. This enables abundances for the remaining molecular species to be determined. The derived abundances generally vary between the sources by no more than a factor of five indicating that circumstellar envelopes around carbon stars with high mass-loss rates have similar chemical compositions. However, there are some notable exceptions. The most striking difference between the abundances are reflecting the spread in the ${ }^{12} \mathrm{C} /{ }^{13} \mathrm{C}$-ratio of about an order of magnitude between the sample stars, which mainly shows the results of nucleosynthesis. The abundance of $\mathrm{SiO}$ also shows a variation of more than an order of magnitude between the sources and is on average more than an order of magnitude more abundant than predicted from photospheric chemistry in thermal equilibrium. The over-abundance of $\mathrm{SiO}$ is consistent with dynamical modelling of the stellar atmosphere and the inner parts of the wind where a pulsation-driven shock has passed. This scenario is possibly further substantiated by the relatively low amount of CS present in the envelopes. The chemistry occurring in the outer envelope is consistent with current photochemical models.
\end{abstract}

Key words. molecular processes - stars: abundances - stars: AGB and post-AGB - stars: carbon - circumstellar matter

\section{Introduction}

The chemistry associated with carbon stars has long been known to be rich and complex in comparison to the alternative $\mathrm{O}$-rich regime (i.e., where $\mathrm{C} / \mathrm{O}<1$ ). This is in part due to the favourable bonding of the carbon atom, enabling long chains and complex species to form. Most of the current understanding of carbon stars has come from both observational and theoretical work on the high-mass-losing carbon star, IRC +10216 . This source, which lies within $200 \mathrm{pc}$ and presents an ideal specimen for the study of carbon-rich envelopes, has been mapped interferometrically in various molecular species (e.g. Bieging \& Tafalla 1993; Dayal \& Bieging 1993, 1995; Gensheimer et al. 1995; Guelin et al. 1993, 1996; Lucas et al. 1995; Lucas \& Guélin 1999) and has had models of its dust (a good summary is given by Men'shchikov et al. 2001) and chemistry (e.g. Millar et al. 2000) constructed. These tools have produced groundbreaking results and have been used to set a paradigm for what has come to be known as "carbon chemistry" in connection with evolved stars. However, the accuracy of employing IRC +10216 chemistry to similar carbon stars has been little-tested due to the difficulties in observing them. Much work has been done on the carbon-rich

\footnotetext{
Send offprint requests to: P. M. Woods, e-mail: pwoods@eso.org

* Figures A.1-A.13 are only available in electronic form at http://www.edpsciences.org
}

post-AGB sources CRL 618 and CRL 2688, and the chemistry of CRL 618 in particular has been modelled by Woods et al. (2003). Detailed chemical studies of carbon stars on the AGB have been few in number, but examples include the molecular line survey of IRAS $15194-5115$, a peculiar ${ }^{13} \mathrm{C}$-rich star (Nyman et al. 1993). Carbon star surveys which include molecular-line comparisons are fewer, and have been limited in the number of lines observed. Olofsson et al. (1993a) detected some 40 stars in a handful of species other than CO. The sample of Bujarrabal et al. (1994) included 16 carbon stars, with up to ten molecular lines observed in each. A more recent survey by Olofsson et al. (1998) detected 22 carbon stars in up to 6 molecular lines.

The survey work presented here purports to be the most complete and consistent molecular-line survey in AGB carbon stars to date, covering high mass-loss rate objects in both the northern and the southern sky. Previously unpublished spectra of five stars (IRAS 15082-4808, IRAS 07454-7112, CIT 6, AFGL 3068 and IRC +40540) are presented, and spectra taken towards IRC +10216 and IRAS 15194-5115 with the SwedishESO Submillimetre Telescope (SEST; Nyman et al. 1993) and IRC +10216 with the Onsala Space Observatory (OSO) $20 \mathrm{~m}$ telescope are used to supplement the survey. Comparison of data from IRC +10216 taken with both telescopes affords a high degree of confidence in the relative calibration that can be derived. 
Table 1. Positions, luminosities, periods and calculated distances of the sample of carbon stars.

\begin{tabular}{lllcccccc}
\hline \hline IRAS No. & Other cat. name & B1950 co-ords. & $\begin{array}{c}P \\
{[\text { days }]}\end{array}$ & $\begin{array}{c}L \\
{\left[L_{\odot}\right]}\end{array}$ & $\begin{array}{c}D \\
{[\mathrm{pc}]}\end{array}$ & $\begin{array}{c}T_{*} \\
{[\mathrm{~K}]}\end{array}$ & $\begin{array}{c}T_{\mathrm{d}} \\
{[\mathrm{K}]}\end{array}$ & $L_{\mathrm{d}} / L_{*}$ \\
\hline $07454-7112$ & AFGL 4078 & $07: 45: 25.7-71: 12: 18$ & - & $9000^{a}$ & 710 & 1200 & 710 & 4.3 \\
$09452+1330$ & IRC +10216 & $09: 45: 15.0+13: 30: 45$ & 630 & 9600 & 120 & - & 510 & - \\
$10131+3049$ & CIT 6 & $10: 13: 11.5+30: 49: 17$ & 640 & 9700 & 440 & 1300 & 510 & 6.7 \\
$15082-4808$ & AFGL 4211 & $15: 08: 13.0-48: 08: 43$ & - & $9000^{a}$ & 640 & - & 590 & - \\
$15194-5115$ & - & $15: 19: 26.9-51: 15: 19$ & 580 & 8800 & 600 & 930 & 480 & 2.2 \\
$23166+1655$ & AFGL 3068 & $23.16 .42 .4+16: 55: 10$ & 700 & 7800 & 820 & - & 1000 & - \\
$23320+4316$ & IRC +40540 & $23: 32: 00.4+43: 16: 17$ & 620 & 9400 & 630 & 1100 & 610 & 6.6 \\
\hline
\end{tabular}

${ }^{a}$ Assumed value.

Up to 51 molecular lines were observed in the sample of 7 high-mass-losing carbon stars, of which 47 were clearly detected. Mass-loss rates, dust properties and the ${ }^{12} \mathrm{CO} /{ }^{13} \mathrm{CO}-$ ratio are calculated self-consistently using a radiative transfer method (Schöier \& Olofsson 2000, 2001; Schöier et al. 2002). An approach similar to that of Nyman et al. (1993) is adopted to calculate fractional abundances (including upper limits), and a detailed analysis of the comparison between the calculated abundances is carried out. Hence, Sect. 2 details the observations carried out and the instrumentation used. Section 3 gives the observational results, including a presentation of various spectra. Section 4 details the NLTE radiative transfer code used to determine the envelope parameters and Sect. 5 explains the method of calculating chemical abundances. The results and deductions are discussed in Sect. 6.

\section{Observations}

\subsection{Carbon star sample}

Following the CO survey of Nyman et al. (1992), several carbon stars, which were bright in $\mathrm{CO}$ lines, were selected for a more comprehensive molecular line search. These stars are rare in that they are all losing mass at a very high rate, and hence are more likely to produce strong emission from a variety of molecular lines. The sample of seven carbon stars (Table 1) was observed using both the $15 \mathrm{~m}$ Swedish-ESO Submillimetre Telescope (Booth et al. 1989) during the period 1987-1996, and the Onsala $20 \mathrm{~m}$ telescope, in 1994. The SEST, situated on La Silla, Chile, was used to observe IRAS 074547112, IRAS 15082-4808, and IRAS 15194-5115. The $20 \mathrm{~m}$ telescope, located at the Onsala Space Observatory (OSO) in Sweden, observed the remaining three sources, CIT 6, AFGL 3068 and IRC +40540 . Both telescopes were used to observe the well-studied carbon star, IRC +10216 in order to determine the relative calibration between the two telescopes.

The JCMT public archive ${ }^{1}$ was searched for complementary line observations, in particular the $\mathrm{CO}$ lines used in the radiation transfer modelling described in Sect. 4. Lines for

\footnotetext{
${ }^{1}$ http://www.jch.hawaii.edu/JACpublic/JCMT/

The JCMT is operated by the Joint Astronomy Centre in Hilo, Hawaii on behalf of the present organisations: the Particle Physics and Astronomy Research Council in The UK, the National Research Council of Canada and The Netherlands Organization for Scientific Research.
}

which multi-epoch observations are available in the JCMT archive typically display intensities that are consistent to 20\% (Schöier \& Olofsson 2001). In addition, interferometric observations of the $\operatorname{CO}(J=1-0)$ brightness distribution around some of the sample stars have been performed (Neri et al. 1998) using the Plateu de Bure interferometer (PdBI), France. The data are publically available and have been used in this paper.

The coordinates used for each individual source are listed in Table 1. Also shown in Table 1 are the adopted luminosities and distances to be used in the molecular excitation analysis. For stars where a period has been determined (see Table 1) the period-luminosity relation from Groenewegen \& Whitelock (1996) was used to estimate the corresponding luminosity. If a reliable period is not available the total bolometric luminosity was fixed to $9000 L_{\odot}$. The distance was then obtained from the luminosity using the observed bolometric magnitude. Schöier \& Olofsson (2001) used the same approach when determining the distances to a large sample of optically bright carbon stars and concluded that there were no apparent systematic effect when comparing with estimates based upon Hipparcos parallaxes, although the scatter is large and the distance estimate for an individual source is subject to some uncertainty of up to a factor of $\sim 2$. The effects of the adopted distance on the molecular excitation will be addressed in Sect. 5.5.

If, for simplicity, the central ratiation field is represented by one or two blackbodies their properties can be determined from a fit to the observed spectral energy distribution (SED) as described in Kerschbaum (1999). A fit to the SED gives the two blackbody temperatures $\left(T_{*}\right.$ and $\left.T_{\mathrm{d}}\right)$ as well as their relative luminosities $\left(L_{\mathrm{d}} / L_{*}\right)$. The parameters obtained in this fashion are presented in Table 1.

\subsection{Instrumentation}

The SEST is equipped with two acousto-optical spectrometers (HRS, $86 \mathrm{MHz}$ bandwidth with $43 \mathrm{kHz}$ channel separation and $80 \mathrm{kHz}$ resolution; LRS, $500 \mathrm{MHz}$ bandwidth with $0.7 \mathrm{MHz}$ channel separation and a resolution of $1.4 \mathrm{MHz}$ ). The receivers used were dual polarization Schottky receivers at both 3 and $1.3 \mathrm{~mm}$ wavelength. Typical system temperatures above the atmosphere were $400-500 \mathrm{~K}$ and $1000-1800 \mathrm{~K}$, respectively.

The OSO $20 \mathrm{~m}$ telescope uses two filterbanks (MUL B, $64 \mathrm{MHz}$ bandwidth with a channel width of $250 \mathrm{kHz}$; MUL A, 
Table 2. Beam widths and efficiencies at selected frequencies.

\begin{tabular}{cccccc}
\hline \hline & \multicolumn{2}{c}{ SEST $15 \mathrm{~m}$} & & \multicolumn{2}{c}{ Onsala $20 \mathrm{~m}$} \\
\cline { 2 - 3 } \cline { 5 - 6 } $\begin{array}{c}\text { Frequency } \\
\text { [GHz] }\end{array}$ & $\begin{array}{c}F W H M \\
{\left[{ }^{\prime \prime}\right]}\end{array}$ & $\eta_{\mathrm{mb}}$ & & $\begin{array}{c}F W M \\
{\left[{ }^{\prime \prime}\right]}\end{array}$ & $\eta_{\mathrm{mb}}$ \\
\hline 86 & 57 & 0.75 & & 44 & 0.58 \\
100 & 51 & 0.73 & & 39 & 0.53 \\
115 & 45 & 0.70 & & 33 & 0.48 \\
230 & 23 & 0.50 & & - & - \\
265 & 21 & 0.42 & & - & - \\
\hline
\end{tabular}

$512 \mathrm{MHz}$ bandwidth and a channel width of $1 \mathrm{MHz}$ ). The receiver used was a horizontally, linearly polarised SIS receiver with a typical system temperature of $400-500 \mathrm{~K}$ above the atmosphere.

All observations were performed using the dual beam switching method, which places the source alternately in two beams, and yields very flat baselines. Beam separation was in both cases about 11'5. Calibration was done with the standard chopper-wheel method. The intensity scales of the spectra are given in main-beam brightness temperature (the corrected antenna temperature, $T_{\mathrm{A}}^{*}$, divided by the main-beam efficiency, $\left.\eta_{\mathrm{mb}}\right)$. Main-beam efficiencies and $F W H M$ beam widths are given in Table 2 for both telescopes.

\section{Observational results}

\subsection{Observed lines}

A total of 196 lines were detected in the sample. 47 transitions of 24 molecular species were detected, and upper limits for another 95 relevant transitions were also obtained, including another three species $\left(\mathrm{C}_{2} \mathrm{~S}, \mathrm{C}_{3} \mathrm{~S}\right.$ and $\left.\mathrm{SO}\right)$. Previously unpublished spectra are shown in Figs. 1, 2 and A.1-A.13. Table 3 lists the detections in all seven sources, together with their peak and integrated intensities. If a line has a hyperfine structure, the frequency and intensity of the strongest component is listed, and the integrated intensity is the sum over all hyperfine components. Values of $T_{\mathrm{mb}}$ for lines where no detection was made are the rms noise values.

Almost all lines observed in IRC +10216 by the SEST were observed in the same source with the OSO $20 \mathrm{~m}$ telescope. The majority of those which were not are due to the lack of a $1.3 \mathrm{~mm}$ receiver at OSO. A large proportion of these lines were observed in the remaining six sources. The purpose of observing IRC +10216 twice, with different telescopes, was to ascertain the relative calibration between the two different setups, and hence gain a basis from which good comparisons could be made between the entire sample of carbon stars.

The ${ }^{13} \mathrm{CS}(J=2-1)$ line observed in some of these sources is partially blended with the $\mathrm{C}_{3} \mathrm{~S}(J=16-15)$ line (Kahane et al. 1988). The ${ }^{13} \mathrm{CS}(J=2-1)$ integrated intensities include both these lines. It is assumed that the relative intensities of these two lines are constant in all the objects, and hence will not affect comparisons. The lines of two $\mathrm{HC}_{3} \mathrm{~N}$ isotopes, $\mathrm{HC}^{13} \mathrm{CCN}$ and $\mathrm{HCC}^{13} \mathrm{CN}$, lie very closely and are in all cases blended together. No attempt is made to separate the components.

\subsection{Line profiles}

There are four characteristic line profile shapes which give information on the source being observed. These lineshapes are most typically seen in $\mathrm{CO}$ emission since it is often strongest (for a comprehensive review of line profiles see Olofsson et al. 1993b). For optically thick emission the line profile can be described as parabolic for unresolved sources or flat-topped for resolved sources. A parabolic profile is shown by most of the ${ }^{12} \mathrm{CO}$ emission lines in the sample except in IRC +10216 and IRAS 15194-5115, where the CO $(J=1-0)$ emission shows a flat-topped profile.

When emission is optically thin, a flat-topped profile is seen for unresolved sources, and a double-peaked profile for a resolved source. Examples of these profiles can be seen in the ${ }^{13} \mathrm{CO}$ emission towards IRAS $15082-4808$ and IRC +10216 . In the following calculations, all lines are assumed optically thin, except those from $\mathrm{CO}$ and $\mathrm{HCN}$.

The values of the expansion velocity, $v_{\text {exp }}$, of the circumstellar envelopes quoted in Table 4 are obtained from the radiative transfer CO modelling of these sources (presented in Sect. 4) where its value is adjusted until a good fit to the observed line profiles is obtained. No trends in the widths of the observed lines are present which lends further support to the assumption adopted here that these envelopes are expanding at constant velocities.

\subsection{Upper limits}

The integrated intensities for lines which were not detected are determined using

$I_{\mathrm{v}} \leq 3 \sigma\left(\sqrt{2} \sqrt{2 \frac{v_{\text {exp }}}{\Delta v_{\text {res }}}}\right) \Delta v=3 \sigma(2 n)^{1 / 2} \Delta v$

where $\sigma$ is the rms noise in the spectrum, $\Delta v$ is the frequency resolution of the spectrum, $v_{\text {res }}$ the velocity resolution of the spectrum and $n$ the number of channels covering the line width. Integrated intensities calculated with this method were used to determine upper limits to abundances.

\section{Full radiative transfer modelling}

In order to obtain accurate values of the mass-loss rates for the objects in the sample, detailed non-LTE radiative transfer modelling of the CO line emission was carried out. This modelling has proven to be one of the most reliable methods for estimating many of the parameters characterising a circumstellar envelope, in particular, the mass-loss rate, kinetic temperature structure, and expansion velocity (Kastner 1992; Groenewegen 1994; Crosas \& Menten 1997; Schöier \& Olofsson 2001; Schöier et al. 2002; Olofsson et al. 2002). Once the basic envelope parameters are known (as well as the stellar parameters), the abundances of various molecules present in the circumstellar envelopes can be determined. 

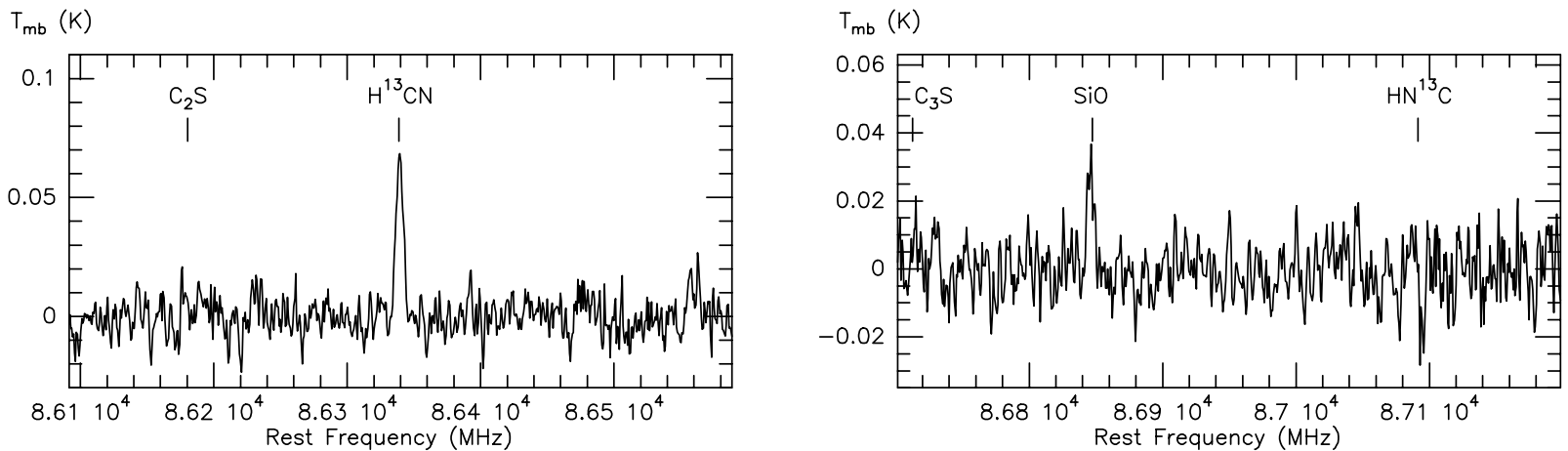

$\mathrm{T}_{\mathrm{mb}}(\mathrm{K})$

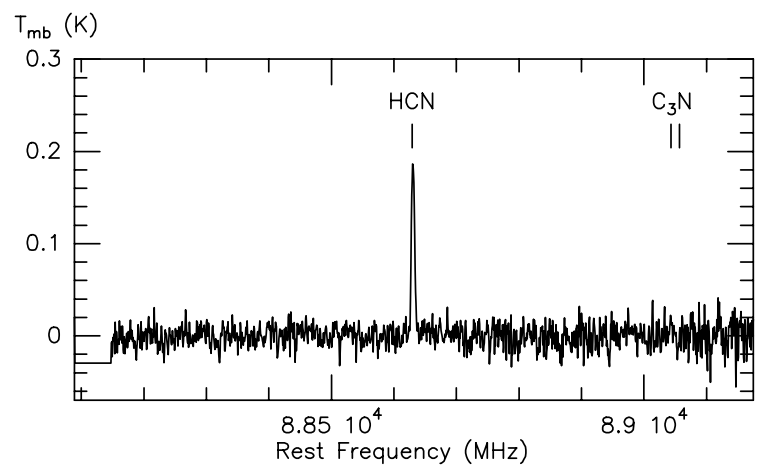

$T_{m b}(K)$

$T_{m b}(K)$
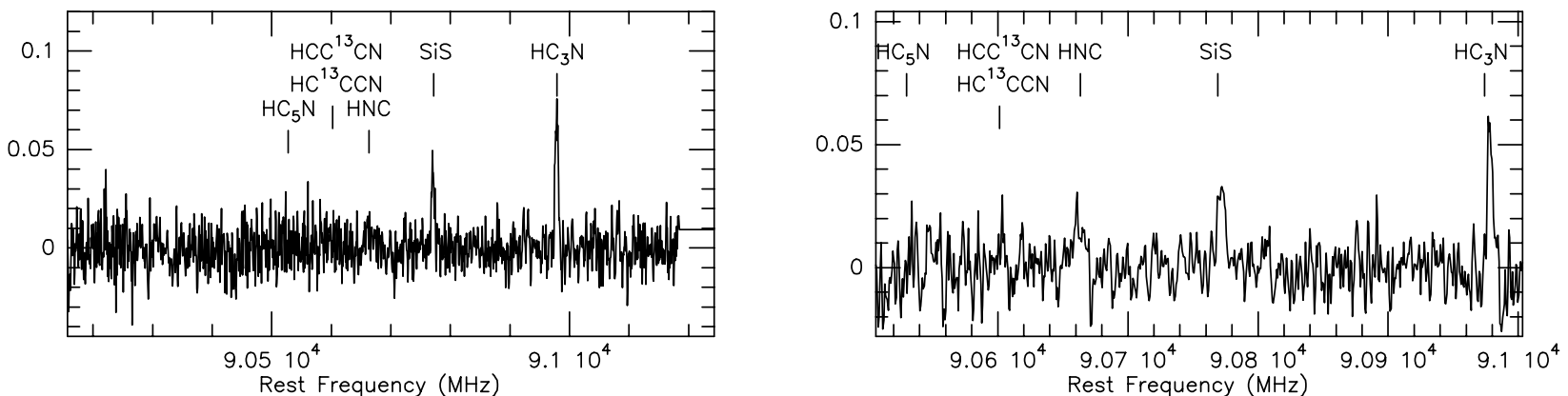

$T_{m b}(K)$

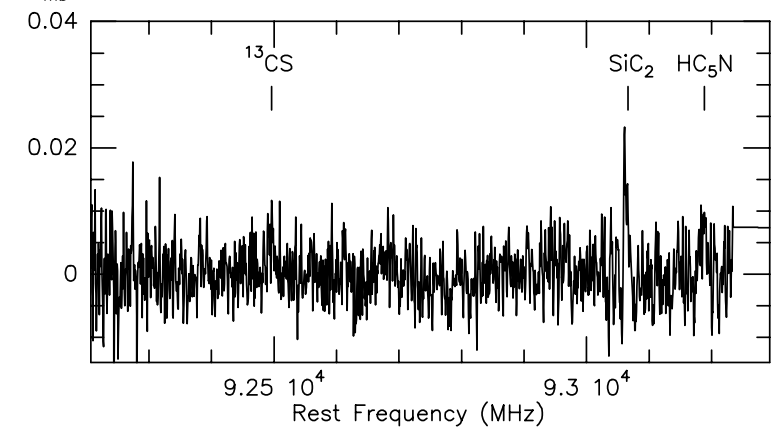

$T_{m b}(K)$

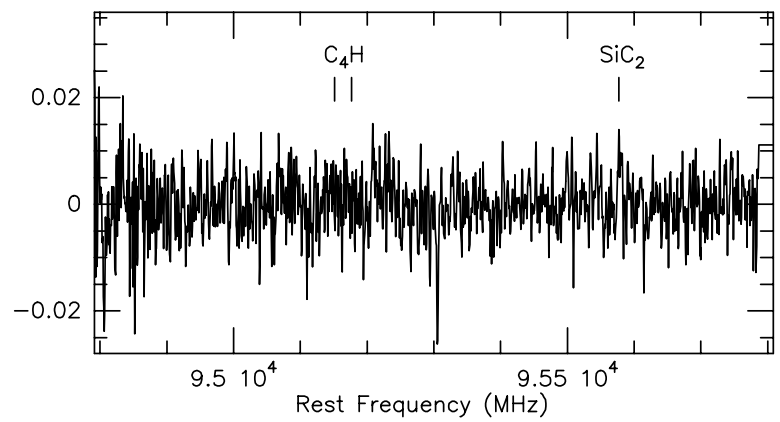

$\mathrm{T}_{\mathrm{mb}}(\mathrm{K})$

$T_{m b}(K)$
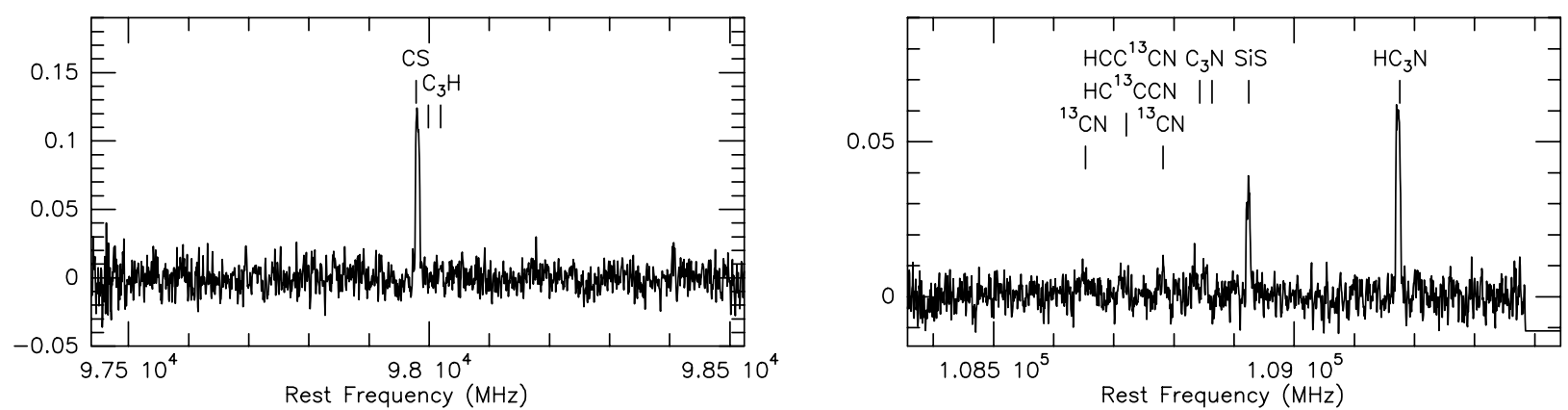

Fig. 1. Low-resolution spectra of IRAS 07454-7112, obtained with the SEST. 
$T_{m b}(K)$

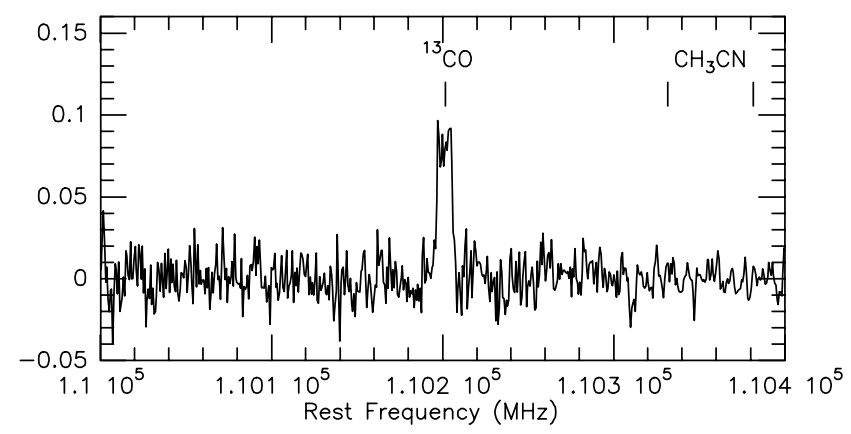

$T_{m b}(K)$

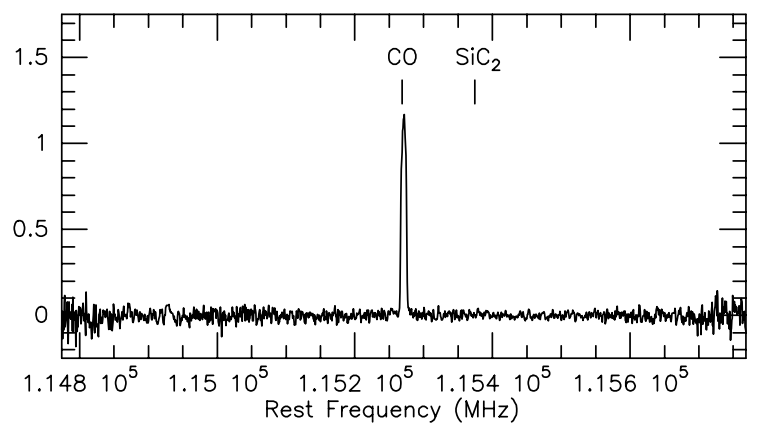

$T_{m b}(K)$

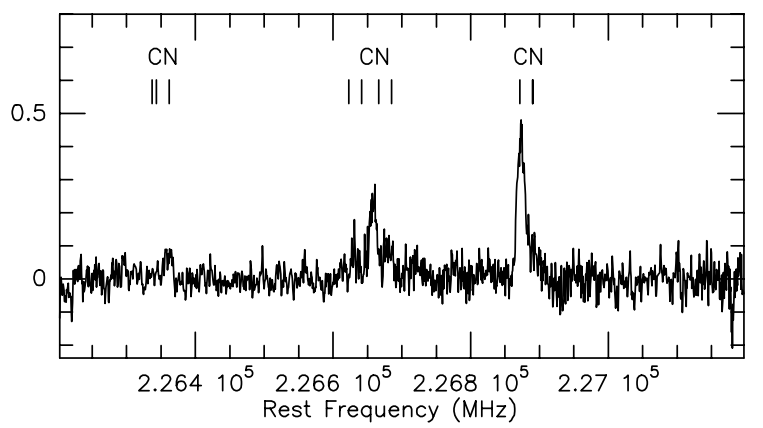

$T_{m b}(K)$

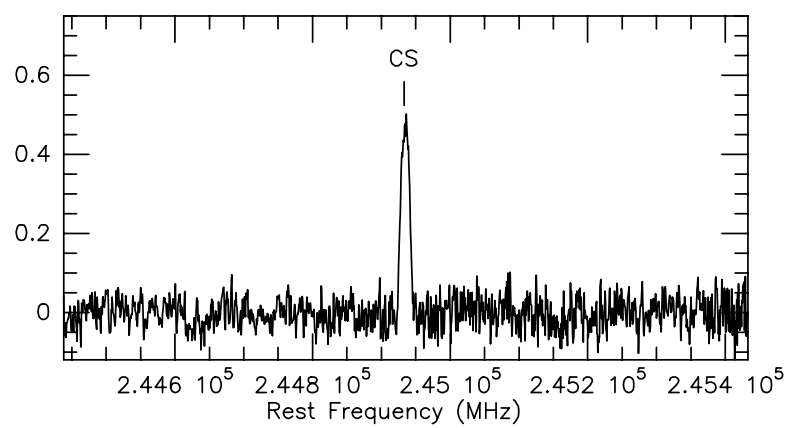

$T_{m b}(K)$

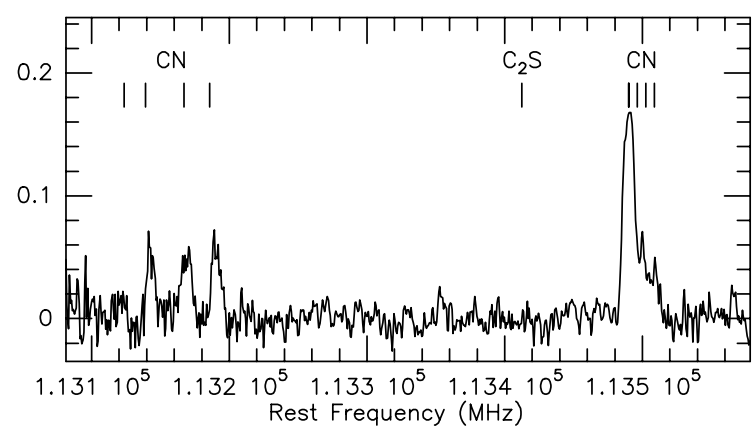

$T_{m b}(K)$

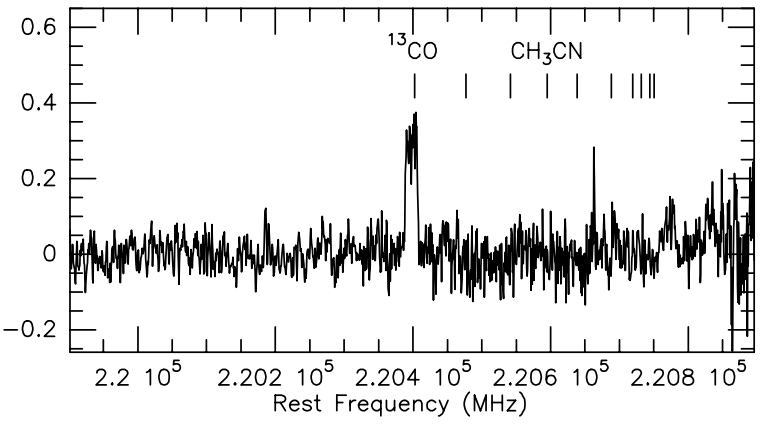

$\mathrm{T}_{\mathrm{mb}}(\mathrm{K})$

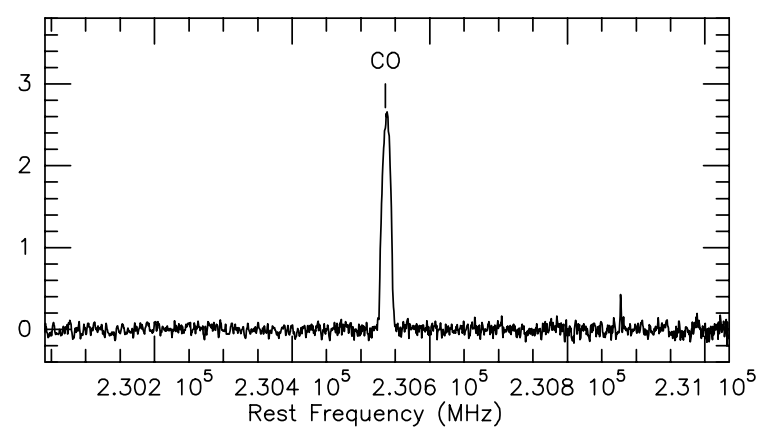

$T_{m b}(K)$

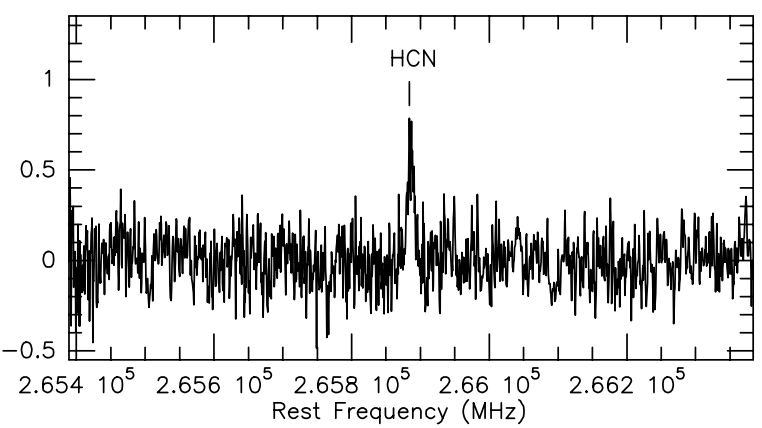

Fig. 2. Low-resolution spectra of IRAS 07454-7112, obtained with the SEST.

\subsection{CO line modelling}

Here the detailed non-LTE radiative transfer code of Schöier (2000) is adopted and the modelling procedure as outlined in detail in Schöier \& Olofsson (2001) is used. The code has been tested against a wide variety of molecular line radiative transfer codes, for a number of benchmark problems, to a high accuracy (van Zadelhoff et al. 2002). The Monte Carlo method (Bernes 1979) is used to determine the steady-state level populations of the $\mathrm{CO}$ molecules in the envelope as a function of distance from the star, using the statistical equilibrium equations. In addition, the code simultaneously solves the energy balance equation including the most relevant heating and cooling processes. Heating is dominated by collisions between the dust and gas except in the outermost parts of the envelope where the photoelectric effect effectively heats the envelope. Cooling is generally dominated by molecular line cooling from $\mathrm{CO}$ but adiabatic cooling due to the expansion of the envelope is also important. The excitation analysis allows for a selfconsistent treatment of CO line cooling. 
Table 3. Detected lines.

\begin{tabular}{|c|c|c|c|c|c|c|c|c|c|c|}
\hline \multirow[t]{2}{*}{ Molecule } & \multirow[t]{2}{*}{ Transition } & \multirow{2}{*}{$\begin{array}{r}\text { Frequency } \\
{[\mathrm{MHz}]}\end{array}$} & \multicolumn{2}{|c|}{ IRC + $10216($ SEST) } & \multicolumn{2}{|c|}{ IRC $+10216(\mathrm{OSO})$} & \multicolumn{2}{|c|}{ IRAS 15194-5115 } & \multicolumn{2}{|c|}{ IRAS $15082-4808$} \\
\hline & & & $\begin{array}{r}T_{\mathrm{mb}} \\
{[\mathrm{K}]} \\
\end{array}$ & $\begin{array}{r}\int T_{\mathrm{mb}} \mathrm{d} v \\
{\left[\mathrm{~K} \mathrm{~km} \mathrm{~s}^{-1}\right]}\end{array}$ & $\begin{array}{r}T_{\mathrm{mb}} \\
{[\mathrm{K}]} \\
\end{array}$ & $\begin{array}{r}\int T_{\mathrm{mb}} \mathrm{d} v \\
{\left[\mathrm{~K} \mathrm{~km} \mathrm{~s}^{-1}\right]}\end{array}$ & $\begin{array}{r}T_{\mathrm{mb}} \\
{[\mathrm{K}]} \\
\end{array}$ & $\begin{array}{r}\int T_{\mathrm{mb}} \mathrm{d} v \\
{\left[\mathrm{~K} \mathrm{~km} \mathrm{~s}^{-1}\right]}\end{array}$ & $\begin{array}{r}T_{\mathrm{mb}} \\
{[\mathrm{K}]} \\
\end{array}$ & $\begin{array}{r}\int T_{\mathrm{mb}} \mathrm{d} v \\
{\left[\mathrm{~K} \mathrm{~km} \mathrm{~s}^{-1}\right]}\end{array}$ \\
\hline $\mathrm{HC}_{5} \mathrm{~N}$ & $J=32-31$ & 85201.348 & 0.15 & 4.78 & - & - & $<0.01$ & $<0.13$ & $<0.01$ & $<0.10$ \\
\hline $\mathrm{C}_{3} \mathrm{H}_{2}$ & $2_{1,2}-1_{0,1}$ & 85338.905 & 0.11 & 2.49 & - & - & 0.02 & 0.84 & $<0.01$ & $<0.10$ \\
\hline $\mathrm{C}_{4} \mathrm{H}$ & $N=9-8, J=19 / 2-17 / 2$ & 85634.00 & 0.20 & 9.60 & - & - & - & - & $<0.01$ & $<0.05$ \\
\hline $\mathrm{C}_{4} \mathrm{H}$ & $N=9-8, J=17 / 2-15 / 2$ & 85672.57 & 0.20 & 9.60 & - & - & - & - & $<0.01$ & $<0.05$ \\
\hline $\mathrm{C}_{2} \mathrm{~S}$ & $6(7)-5(6)$ & 86181.413 & $<0.03$ & $<0.46$ & $<0.04$ & $<0.76$ & $<0.07$ & $<0.11$ & $<0.04$ & $<0.06$ \\
\hline $\mathrm{H}^{13} \mathrm{CN}$ & $J=1-0$ & 86340.184 & 3.23 & 87.20 & 3.86 & 114.14 & 0.57 & 20.93 & 0.09 & 3.06 \\
\hline $\mathrm{C}_{3} \mathrm{~S}$ & $J=15-14$ & 86708.379 & $<0.02$ & $<0.30$ & $<0.03$ & $<0.65$ & $<0.01$ & $<0.08$ & $<0.01$ & $<0.06$ \\
\hline $\mathrm{SiO}$ & $J=2-1$ & 86846.998 & 0.71 & 20.40 & 0.95 & 24.48 & 0.16 & 5.33 & 0.07 & 1.84 \\
\hline $\mathrm{HN}^{13} \mathrm{C}$ & $J=1-0$ & 87090.859 & $<0.04$ & $<0.62$ & - & - & 0.02 & 1.11 & $<0.01$ & $<0.11$ \\
\hline $\mathrm{C}_{2} \mathrm{H}$ & $N=1-0$ & 87316.925 & 0.52 & 41.60 & 0.66 & 49.48 & 0.13 & 11.50 & 0.04 & 4.04 \\
\hline $\mathrm{HCN}$ & $J=1-0$ & 88631.847 & 7.80 & 190.93 & 9.14 & 214.04 & 0.55 & 15.33 & 0.37 & 11.26 \\
\hline $\mathrm{C}_{3} \mathrm{~N}$ & $N=9-8, J=19 / 2-17 / 2$ & 89045.59 & 0.31 & 9.19 & - & - & $<0.01$ & $<0.07$ & $<0.01$ & $<0.09$ \\
\hline $\mathrm{C}_{3} \mathrm{~N}$ & $N=9-8, J=17 / 2-15 / 2$ & 89064.36 & 0.31 & 9.19 & - & - & $<0.01$ & $<0.07$ & $<0.01$ & $<0.09$ \\
\hline $\mathrm{HC}_{5} \mathrm{~N}$ & $J=34-33$ & 90525.892 & 0.16 & 2.58 & $<0.03$ & $<0.67$ & - & - & $<0.01$ & $<0.10$ \\
\hline $\mathrm{HC}^{13} \mathrm{CCN}$ & $J=10-9$ & 90593.059 & 0.09 & 1.98 & $<0.03$ & $<0.34$ & 0.02 & 0.76 & $<0.01$ & $<0.05$ \\
\hline $\mathrm{HCC}^{13} \mathrm{CN}$ & $J=10-9$ & 90601.791 & 0.09 & 1.98 & $<0.03$ & $<0.34$ & 0.02 & 0.76 & $<0.01$ & $<0.05$ \\
\hline $\mathrm{HNC}$ & $J=1-0$ & 90663.543 & 0.74 & 26.49 & 0.58 & 17.22 & 0.08 & 3.72 & 0.05 & 1.70 \\
\hline $\mathrm{SiS}$ & $J=5-4$ & 90771.546 & 1.14 & 35.54 & 1.07 & 39.47 & 0.05 & 2.78 & 0.03 & 1.58 \\
\hline $\mathrm{HC}_{3} \mathrm{~N}$ & $J=10-9$ & 90978.993 & 2.42 & 64.59 & 2.00 & 52.63 & 0.09 & 3.71 & 0.11 & 4.15 \\
\hline${ }^{13} \mathrm{CS}$ & $J=2-1$ & 92494.299 & 0.07 & 2.97 & - & - & 0.06 & 2.67 & $<0.01$ & $<0.10$ \\
\hline $\mathrm{SiC}_{2}$ & $4_{0,4}-3_{0,3}$ & 93063.639 & 0.46 & 14.60 & - & - & - & - & 0.03 & 0.40 \\
\hline $\mathrm{HC}_{5} \mathrm{~N}$ & $J=35-34$ & 93188.127 & 0.12 & 5.50 & - & - & - & - & $<0.01$ & $<0.10$ \\
\hline $\mathrm{C}_{4} \mathrm{H}$ & $N=10-9, J=21 / 2-19 / 2$ & 95150.32 & 0.19 & 6.08 & 0.18 & 8.75 & 0.04 & 1.95 & 0.02 & 0.47 \\
\hline $\mathrm{C}_{4} \mathrm{H}$ & $N=10-9, J=19 / 2-17 / 2$ & 95188.94 & 0.19 & 6.08 & 0.18 & 8.75 & 0.04 & 1.95 & 0.02 & 0.47 \\
\hline $\mathrm{SiC}_{2}$ & $4_{2,2}-3_{2,1}$ & 95579.389 & 0.32 & 10.73 & - & - & - & - & $<0.01$ & $<0.08$ \\
\hline $\mathrm{C}^{34} \mathrm{~S}$ & $J=2-1$ & 96412.982 & 0.15 & 5.66 & - & - & - & - & - & - \\
\hline CS & $J=2-1$ & 97980.968 & 2.92 & 81.23 & 4.06 & 118.89 & 0.37 & 15.21 & 0.25 & 8.44 \\
\hline $\mathrm{C}_{3} \mathrm{H}$ & ${ }^{2} \Pi_{1 / 2}, 9 / 2-7 / 2$ & 97995.450 & 0.18 & 11.27 & 0.28 & 14.54 & 0.01 & 0.64 & $<0.01$ & $<0.11$ \\
\hline so & $J=3-2$ & 99299.879 & $<0.02$ & $<0.25$ & - & - & - & - & - & - \\
\hline $\mathrm{HC}_{3} \mathrm{~N}$ & $J=11-10$ & 100076.389 & 2.21 & 53.84 & - & - & - & - & - & - \\
\hline $\mathrm{HC}^{13} \mathrm{CCN}$ & $J=12-11$ & 108710.523 & 0.09 & 2.34 & 0.14 & 1.22 & 0.01 & 0.39 & $<0.01$ & $<0.03$ \\
\hline $\mathrm{HCC}^{13} \mathrm{CN}$ & $J=12-11$ & 108721.008 & 0.09 & 2.34 & 0.14 & 1.22 & 0.01 & 0.39 & $<0.01$ & $<0.03$ \\
\hline${ }^{13} \mathrm{CN}$ & $N=1-0$ & 108780.201 & 0.11 & 8.56 & 0.24 & 6.07 & 0.02 & 2.18 & $<0.01$ & $<0.05$ \\
\hline $\mathrm{C}_{3} \mathrm{~N}$ & $N=11-10, J=23 / 2-21 / 2$ & 108834.27 & 0.39 & 11.76 & 0.64 & 18.00 & 0.01 & 0.68 & 0.02 & 0.63 \\
\hline $\mathrm{C}_{3} \mathrm{~N}$ & $N=11-10, J=21 / 2-19 / 2$ & 108853.02 & 0.39 & 11.76 & 0.64 & 18.00 & 0.01 & 0.68 & 0.02 & 0.63 \\
\hline SiS & $J=6-5$ & 108924.267 & 1.10 & 30.00 & 1.86 & 52.40 & 0.05 & 2.44 & 0.04 & 1.14 \\
\hline $\mathrm{HC}_{3} \mathrm{~N}$ & $J=12-11$ & 109173.634 & 2.03 & 52.39 & - & - & 0.07 & 3.16 & 0.08 & 2.79 \\
\hline $\mathrm{C}^{18} \mathrm{O}$ & $J=1-0$ & 109782.160 & 0.02 & 0.76 & - & - & - & - & $<0.01$ & $<0.17$ \\
\hline${ }^{13} \mathrm{CO}$ & $J=1-0$ & 110201.353 & 1.69 & 26.62 & 2.50 & 36.40 & 0.31 & 14.93 & 0.06 & 1.82 \\
\hline $\mathrm{CH}_{3} \mathrm{CN}$ & $6(1)-5(1)$ & 110381.404 & 0.09 & 4.77 & $<0.05$ & $<1.42$ & $<0.01$ & $<0.18$ & $<0.01$ & $<0.23$ \\
\hline $\mathrm{C}_{2} \mathrm{~S}$ & $8(9)-7(8)$ & 113410.207 & $<0.03$ & $<0.48$ & $<0.07$ & $<1.96$ & $<0.02$ & $<0.53$ & $<0.01$ & $<0.11$ \\
\hline $\mathrm{CN}$ & $N=1-0$ & 113490.982 & 3.43 & 238.43 & 3.88 & 310.20 & 0.13 & 16.43 & 0.19 & 14.71 \\
\hline $\mathrm{CO}$ & $J=1-0$ & 115271.204 & 10.29 & 269.43 & 20.83 & 542.71 & 1.27 & 55.86 & 1.11 & 36.71 \\
\hline $\mathrm{SiC}_{2}$ & $5_{0,5}-4_{0,4}$ & 115382.38 & 0.80 & 21.43 & 1.60 & 39.38 & 0.07 & 2.66 & 0.03 & 0.82 \\
\hline $\mathrm{C}^{18} \mathrm{O}$ & $J=2-1$ & 219560.319 & 0.15 & 4.21 & - & - & - & - & - & - \\
\hline${ }^{13} \mathrm{CO}$ & $J=2-1$ & 220398.686 & 3.85 & 79.62 & - & - & 0.90 & 35.58 & 0.15 & 5.76 \\
\hline $\mathrm{CH}_{3} \mathrm{CN}$ & $12(0)-11(0)$ & 220747.268 & 0.17 & 2.80 & - & - & $<0.05$ & $<1.01$ & $<0.03$ & $<0.48$ \\
\hline $\mathrm{CN}$ & $N=2-1$ & 226874.564 & 2.55 & 231.18 & - & - & 0.15 & 17.06 & 0.20 & 11.32 \\
\hline $\mathrm{CO}$ & $J=2-1$ & 230538.000 & 34.60 & 799.00 & - & - & 4.20 & 150.00 & 2.54 & 69.40 \\
\hline CS & $J=5-4$ & 244935.606 & 16.57 & 405.53 & - & - & 0.77 & 32.34 & 0.47 & 10.89 \\
\hline $\mathrm{HCN}$ & $J=3-2$ & 265886.432 & 45.10 & 1010.24 & - & - & 4.69 & 139.76 & 0.88 & 20.83 \\
\hline
\end{tabular}

The observed circumstellar CO line emission is modelled taking into account 50 rotational levels in each of the fundamental and first excited vibrational states. The energy levels and radiative transition probabilities from Chandra et al. (1996) are used. The recently published collisional rates of $\mathrm{CO}$ with $\mathrm{H}_{2}$ by Flower (2001) have been adopted assuming an ortho-to-para ratio of 3 . For temperatures above $400 \mathrm{~K}$ the rates from Schinke et al. (1985) were used and further extrapolated to include transitions up to $J=50$. The collisional rates adopted here differ from those used in the previous modelling of some of these sources (Ryde et al. 1999; Schöier \& Olofsson 2000; Schöier \& Olofsson 2001) and account for the slightly different envelope parameters derived in the present analysis.
The same set of collisional rates were used for all $\mathrm{CO}$ isotopomers.

The envelopes are assumed to be spherically symmetric and to expand at a constant velocity and the model includes the radiation emitted from the central star. Dust present around the star will absorb parts of the stellar radiation and re-emit it at longer wavelenghts. For simplicity, the central radiation field is represented by one or two blackbodies and is determined from a fit to the observed spectral energy distribution (SED) (Table 1). The inner radius of the circumstellar envelope is taken to reside outside that of the central blackbodies. This procedure provides a good description of the radiation field to which the envelope is subjected. For the sample stars, which 
Table 3. continued.

\begin{tabular}{|c|c|c|c|c|c|c|c|c|c|c|}
\hline \multirow[t]{2}{*}{ Molecule } & \multirow[t]{2}{*}{ Transition } & \multirow{2}{*}{$\begin{array}{r}\text { Frequency } \\
(\mathrm{MHz})\end{array}$} & \multicolumn{2}{|c|}{ IRAS 07454-7112 } & \multicolumn{2}{|c|}{ CIT 6} & \multicolumn{2}{|c|}{ AFGL 3068} & \multicolumn{2}{|c|}{ IRC +40540} \\
\hline & & & $\begin{array}{l}T_{\mathrm{mb}} \\
(\mathrm{K})\end{array}$ & $\begin{array}{r}\int T_{\mathrm{mb}} \mathrm{d} v \\
\left(\mathrm{~K} \mathrm{~km} \mathrm{~s}^{-1}\right)\end{array}$ & $\begin{array}{r}T_{\mathrm{mb}} \\
(\mathrm{K})\end{array}$ & $\begin{array}{r}\int T_{\mathrm{mb}} \mathrm{d} v \\
\left(\mathrm{~K} \mathrm{~km} \mathrm{~s}^{-1}\right)\end{array}$ & $\begin{array}{r}T_{\mathrm{mb}} \\
(\mathrm{K})\end{array}$ & $\begin{array}{r}\int T_{\mathrm{mb}} \mathrm{d} v \\
\left(\mathrm{~K} \mathrm{~km} \mathrm{~s}^{-1}\right)\end{array}$ & $\begin{array}{r}T_{\mathrm{mb}} \\
(\mathrm{K})\end{array}$ & $\begin{array}{r}\int T_{\mathrm{mb}} \mathrm{d} v \\
\left(\mathrm{Kkm} \mathrm{s}^{-1}\right)\end{array}$ \\
\hline $\mathrm{C}_{2} \mathrm{~S}$ & $6(7)-5(6)$ & 86181.413 & $<0.01$ & $<0.07$ & $<0.02$ & $<0.36$ & $<0.02$ & $<0.44$ & $<0.01$ & $<0.28$ \\
\hline $\mathrm{H}^{13} \mathrm{CN}$ & $J=1-0$ & 86340.184 & 0.07 & 1.45 & 0.15 & 3.13 & 0.13 & 3.76 & 0.13 & 3.85 \\
\hline $\mathrm{C}_{3} \mathrm{~S}$ & $J=15-14$ & 86708.379 & $<0.01$ & $<0.07$ & $<0.01$ & $<0.23$ & $<0.02$ & $<0.47$ & $<0.01$ & $<0.21$ \\
\hline $\mathrm{SiO}$ & $J=2-1$ & 86846.998 & 0.03 & 0.52 & 0.17 & 3.16 & $<0.02$ & $<0.47$ & 0.04 & 0.94 \\
\hline $\mathrm{HN}^{13} \mathrm{C}$ & $J=1-0$ & 87090.859 & $<0.01$ & $<0.08$ & $<0.01$ & $<0.23$ & $<0.02$ & $<0.47$ & $<0.01$ & $<0.25$ \\
\hline $\mathrm{C}_{2} \mathrm{H}$ & $N=1-0$ & 87316.925 & $<0.01$ & $<0.09$ & 0.10 & 5.81 & 0.09 & 5.94 & $<0.01$ & $<1.78$ \\
\hline $\mathrm{HCN}$ & $J=1-0$ & 88631.847 & 0.19 & 4.43 & 0.77 & 18.07 & 0.39 & 8.97 & 0.26 & 8.24 \\
\hline $\mathrm{C}_{3} \mathrm{~N}$ & $N=9-8, J=19 / 2-17 / 2$ & 89045.59 & $<0.01$ & $<0.07$ & - & - & - & - & - & - \\
\hline $\mathrm{C}_{3} \mathrm{~N}$ & $N=9-8, J=17 / 2-15 / 2$ & 89064.36 & $<0.01$ & $<0.06$ & - & - & - & - & - & - \\
\hline $\mathrm{HC}_{5} \mathrm{~N}$ & $J=34-33$ & 90525.892 & 0.02 & 0.06 & $<0.01$ & $<0.21$ & $<0.01$ & $<0.21$ & $<0.02$ & $<0.37$ \\
\hline $\mathrm{HC}^{13} \mathrm{CCN}$ & $J=10-9$ & 90593.059 & 0.01 & 0.13 & $<0.01$ & $<0.11$ & $<0.01$ & $<0.10$ & 0.08 & 0.35 \\
\hline $\mathrm{HCC}^{13} \mathrm{CN}$ & $J=10-9$ & 90601.791 & 0.01 & 0.13 & $<0.01$ & $<0.11$ & $<0.01$ & $<0.10$ & 0.08 & 0.35 \\
\hline $\mathrm{HNC}$ & $J=1-0$ & 90663.543 & 0.02 & 0.50 & 0.13 & 2.95 & 0.06 & 1.18 & 0.09 & 1.42 \\
\hline SiS & $J=5-4$ & 90771.546 & 0.03 & 1.07 & 0.04 & 0.59 & 0.04 & 0.59 & 0.15 & 4.11 \\
\hline $\mathrm{HC}_{3} \mathrm{~N}$ & $J=10-9$ & 90978.993 & 0.06 & 1.39 & 0.19 & 5.17 & 0.12 & 3.37 & 0.19 & 6.16 \\
\hline${ }^{13} \mathrm{CS}$ & $J=2-1$ & 92494.299 & $<0.01$ & $<0.04$ & - & - & - & - & - & - \\
\hline $\mathrm{SiC}_{2}$ & $4_{0,4}-3_{0,3}$ & 93063.639 & 0.01 & 0.13 & - & - & - & - & - & - \\
\hline $\mathrm{HC}_{5} \mathrm{~N}$ & $J=35-34$ & 93188.127 & $<0.01$ & $<0.04$ & - & - & - & - & - & - \\
\hline $\mathrm{C}_{4} \mathrm{H}$ & $N=10-9, J=21 / 2-19 / 2$ & 95150.32 & $<0.01$ & $<0.03$ & $<0.01$ & $<0.13$ & - & - & - & - \\
\hline $\mathrm{C}_{4} \mathrm{H}$ & $N=10-9, J=19 / 2-17 / 2$ & 95188.94 & $<0.01$ & $<0.03$ & $<0.01$ & $<0.13$ & - & - & - & - \\
\hline $\mathrm{SiC}_{2}$ & $4_{2,2}-3_{2,1}$ & 95579.389 & $<0.01$ & $<0.06$ & - & - & - & - & - & - \\
\hline CS & $J=2-1$ & 97980.968 & 0.13 & 2.53 & 0.67 & 16.91 & 0.14 & 3.93 & 0.30 & 9.56 \\
\hline $\mathrm{C}_{3} \mathrm{H}$ & ${ }^{2} \Pi_{1 / 2}, 9 / 2-7 / 2$ & 97995.450 & $<0.01$ & $<0.09$ & $<0.02$ & $<0.55$ & $<0.01$ & $<0.31$ & $<0.02$ & $<0.45$ \\
\hline $\mathrm{HC}^{13} \mathrm{CCN}$ & $J=12-11$ & 108710.523 & 0.01 & 0.08 & 0.05 & 0.44 & $<0.01$ & $<0.14$ & $<0.01$ & $<0.09$ \\
\hline $\mathrm{HCC}^{13} \mathrm{CN}$ & $J=12-11$ & 108721.008 & 0.01 & 0.08 & 0.05 & 0.44 & $<0.01$ & $<0.14$ & $<0.01$ & $<0.09$ \\
\hline${ }^{13} \mathrm{CN}$ & $N=1-0$ & 108780.201 & 0.01 & 0.73 & 0.05 & 4.21 & $<0.01$ & $<0.28$ & $<0.01$ & $<0.18$ \\
\hline $\mathrm{C}_{3} \mathrm{~N}$ & $N=11-10, J=23 / 2-21 / 2$ & 108834.27 & 0.01 & 0.23 & 0.10 & 2.19 & 0.05 & 1.34 & 0.03 & 0.70 \\
\hline $\mathrm{C}_{3} \mathrm{~N}$ & $N=11-10, J=21 / 2-19 / 2$ & 108853.02 & 0.01 & 0.23 & 0.10 & 2.19 & 0.05 & 1.34 & 0.03 & 0.70 \\
\hline SiS & $J=6-5$ & 108924.267 & 0.03 & 0.88 & 0.19 & 3.62 & 0.15 & 2.23 & 0.07 & 1.91 \\
\hline $\mathrm{HC}_{3} \mathrm{~N}$ & $J=12-11$ & 109173.634 & 0.06 & 1.48 & - & - & - & - & - & - \\
\hline${ }^{13} \mathrm{CO}$ & $J=1-0$ & 110201.353 & 0.09 & 2.10 & 0.40 & 5.90 & 0.17 & 9.33 & 0.22 & 4.39 \\
\hline $\mathrm{CH}_{3} \mathrm{CN}$ & $6(1)-5(1)$ & 110381.404 & $<0.01$ & $<0.12$ & $<0.01$ & $<0.29$ & $<0.03$ & $<0.83$ & $<0.03$ & $<0.72$ \\
\hline $\mathrm{C}_{2} \mathrm{~S}$ & $8(9)-7(8)$ & 113410.207 & $<0.01$ & $<0.10$ & $<0.03$ & $<0.89$ & $<0.02$ & $<0.53$ & $<0.02$ & $<0.64$ \\
\hline $\mathrm{CN}$ & $N=1-0$ & 113490.982 & 0.17 & 10.30 & 1.12 & 61.84 & 0.09 & 5.97 & 0.41 & 21.84 \\
\hline $\mathrm{CO}$ & $J=1-0$ & 115271.204 & 1.16 & 25.57 & 3.52 & 105.00 & 2.08 & 47.08 & 1.94 & 41.88 \\
\hline $\mathrm{SiC}_{2}$ & $5_{0,5}-4_{0,4}$ & 115382.38 & $<0.01$ & $<0.24$ & 0.23 & 6.71 & $<0.02$ & $<0.62$ & $<0.03$ & $<0.75$ \\
\hline${ }^{13} \mathrm{CO}$ & $J=2-1$ & 220398.686 & 0.25 & 7.34 & - & - & - & - & - & - \\
\hline $\mathrm{CH}_{3} \mathrm{CN}$ & $12(0)-11(0)$ & 220747.268 & 0.04 & 0.67 & - & - & - & - & - & - \\
\hline $\mathrm{CN}$ & $N=2-1$ & 226874.564 & 0.41 & 20.59 & - & - & - & - & - & - \\
\hline $\mathrm{CO}$ & $J=2-1$ & 230538.000 & 2.60 & 50.00 & - & - & - & - & - & - \\
\hline CS & $J=5-4$ & 244935.606 & 0.49 & 8.12 & - & - & - & - & - & - \\
\hline $\mathrm{HCN}$ & $J=3-2$ & 265886.432 & 0.50 & 8.82 & - & - & - & - & - & - \\
\hline
\end{tabular}

Table 4. Summary of circumstellar properties derived from the CO modelling (see text for details).

\begin{tabular}{|c|c|c|c|c|c|c|c|c|c|}
\hline Source & $\begin{array}{c}\dot{M} \\
{\left[M_{\odot} \mathrm{yr}^{-1}\right]}\end{array}$ & $h$ & $N^{b}$ & $\chi_{\text {red }}^{2}{ }^{c}$ & $\begin{array}{c}v_{\exp } \\
{\left[\mathrm{km} \mathrm{s}^{-1}\right]}\end{array}$ & $\frac{\left(\dot{M} / v_{\text {exp }}\right)_{\star}}{\left(\dot{M} / v_{\text {exp }}\right)_{10216}}$ & $\begin{array}{c}r_{\mathrm{p}}^{d} \\
{[\mathrm{~cm}]}\end{array}$ & $\begin{array}{c}d \\
{[\mathrm{~cm}]}\end{array}$ & $\frac{{ }^{12} \mathrm{CO}^{e}}{{ }^{13} \mathrm{CO}}$ \\
\hline IRAS 07454-7112 & $5.0(-6)$ & $1.5^{a}$ & 2 & 0.1 & 13.0 & 0.46 & $3.0(17)$ & $2.4(16)$ & 17 \\
\hline IRC +10216 & $1.2(-5)$ & 1.5 & 27 & 1.4 & 14.5 & 1.00 & $3.7(17)$ & $5.5(16)$ & 45 \\
\hline CIT 6 & $5.0(-6)$ & 2.3 & 20 & 0.8 & 17.0 & 0.36 & $1.9(17)$ & $2.8(16)$ & 35 \\
\hline IRAS $15082-4808$ & $1.0(-5)$ & $1.5^{a}$ & 2 & 0.2 & 19.5 & 0.62 & $2.5(17)$ & $3.5(16)$ & 35 \\
\hline IRAS $15194-5115$ & $1.2(-5)$ & 2.8 & 10 & 0.9 & 21.5 & 0.67 & $3.2(17)$ & $7.2(16)$ & 6 \\
\hline AFGL 3068 & $2.0(-5)$ & 2.5 & 4 & 1.6 & 14.0 & 1.72 & $3.8(17)$ & $1.2(17)$ & 30 \\
\hline $\mathrm{IRC}+40540$ & $1.5(-5)$ & 1.5 & 7 & 1.0 & 14.0 & 1.29 & $4.0(17)$ & $7.3(16)$ & 50 \\
\hline
\end{tabular}

${ }^{a}$ Adopted value.

${ }^{b}$ Number of observational constraints used in the ${ }^{12} \mathrm{CO}$ modelling.

${ }^{c}$ Reduced $\chi^{2}$ of the best fit ${ }^{12} \mathrm{CO}$ model.

${ }^{d} r_{\mathrm{p}}$ is the photodissociation radius of $\mathrm{CO}$.

${ }^{e}$ Determined from radiative transfer modelling of both ${ }^{12} \mathrm{CO}$ and ${ }^{13} \mathrm{CO}$ emission. 
have dense CSEs, the line intensities derived from the ${ }^{12} \mathrm{CO}$ model are not sensitive to the adopted description of the stellar spectrum due to the high line optical depths. The stellar photons are typically absorbed within the first few shells in the model. A more detailed treatment of thermal emission from the dust present in the CSE, and the increase of total optical depth at the line wavelenghts, has been found to be of no major importance in deriving the envelope parameters for high mass-loss rate objects (Schöier et al. 2002).

The abundance of ${ }^{12} \mathrm{CO}$ relative to $\mathrm{H}_{2}$ was fixed at $1.0 \times$ $10^{-3}$, in agreement with Willacy \& Cherchneff (1998) and the survey of Olofsson et al. (1993a). The CO envelope size was estimated based upon modelling results from Mamon et al. (1988), which have been shown to compare well with observations (Schöier \& Olofsson 2001). The same envelope size was assumed for all $\mathrm{CO}$ isotopomers.

\subsection{Fitting strategy}

With the assumptions made in the standard model there remains two principal free parameters, the mass-loss rate $(\dot{M})$ and the so-called $h$-parameter, which contains information about individual dust grains and controls the amount of heating through dust-gas collisions.

$h=\left(\frac{\Psi}{0.01}\right)\left(\frac{2.0 \mathrm{~g} \mathrm{~cm}^{-3}}{\rho_{\mathrm{d}}}\right)\left(\frac{0.05 \mu \mathrm{m}}{a_{\mathrm{d}}}\right)$,

where $\Psi$ is the dust-to-gas mass-loss rate ratio, $\rho_{\mathrm{d}}$ the mass density of a dust grain, and $a_{\mathrm{d}}$ the size of a dust grain, and the adopted values of these parameters are also given. The massloss rate and the $h$-parameter were allowed to vary simultaneously. Once the molecular excitation, i.e., the level populations, is obtained the radiative transfer equation can be solved exactly. The resulting brightness distribution is then convolved with the appropriate beam to allow a direct comparison with observations. The beam profile used in the convolution of the modelled emission is assumed to be Gaussian which is appropriate at the frequencies used here. The best fit circumstellar model for a particular source is estimated from a $\chi^{2}$-analysis (for details see Schöier \& Olofsson 2001) using observed ${ }^{12} \mathrm{CO}$ integrated intensities and assuming a 15-20\% calibration uncertainty. The number of observational constraints used in the modelling is shown in Table 4 together with the reduced $\chi^{2}$ of the best fit model. In all the cases $\chi_{\text {red }}^{2} \sim 1$, indicating good fits.

The ${ }^{12} \mathrm{CO}$ data used in the analysis are presented in Schöier \& Olofsson (2001) and Schöier et al. (2002) for CIT 6, IRC +10216, IRAS 15194-5115, and IRC +40540 and consist of both millimetre and sub-millimetre line data as well as far infra-red high- $J$ transitions observed by ISO. The best fit ${ }^{12} \mathrm{CO}$ model obtained for AFGL 3068 is overlayed onto observations and presented in Fig. 3. For IRAS 07454-7112 and IRAS $15082-4808$ only $J=1-0$ and $J=2-1$ line data as observed by the SEST, and presented here in Figs. 1, 2, A.1, A.2 and A.6-A.9, are available. Due to the limited number of constraints the $h$-parameter was assumed to be equal to 1.5 , i.e., the dust properties were taken to be the same as for IRC +10216 and IRC +40540 for these two sources.

\subsection{The mass-loss rates}

The determination of accurate mass-loss rates for some of these carbon stars is, it turns out, difficult in models where CO cooling is treated in a self-consistent manner (Sahai 1990; Kastner 1992; Schöier \& Olofsson 2001). The reason for this is that an increase in $\dot{M}$ leads to more net cooling than heating, which compensates for the increase in molecular density, making it hard to simultaneously constrain both $\dot{M}$ and the $h$-parameter. For the sources CIT 6, IRC +10216 and IRAS 15194-5115, where a significant number of high- $J$ transitions were observed by ISO, the degeneracy between $\dot{M}$ and the $h$-parameter is partly lifted allowing for better constraints to be put on these parameters (Schöier et al. 2002). For these three sources the mass-loss rate is estimated to be accurate to about $50 \%$ within the adopted circumstellar model. For the remaining sources the uncertainty in the derived mass-loss rate is larger due to either the above mentioned degeneracy (AFGL 3068 and IRC +40540 ) or that only two ${ }^{12} \mathrm{CO}$ lines are used in the analysis (IRAS 07454-7112 and IRAS 15082-4808). Radiative transfer analysis of the observed continuum emission from these sources (Schöier et al., in prep.) give mass-loss rates that agree within $\sim 50 \%$ when compared with those derived from the $\mathrm{CO}$ modelling. This is reassuring and lends further credibility to the mass-loss rates presented in Table 4.

The CSEs of the sample stars have similar physical properties. However, the stars presented here are losing mass at a significantly higher rate than the average carbon star: Schöier \& Olofsson (2001) measure a median mass-loss rate for carbon stars of $\sim 3 \times 10^{-7} M_{\odot} \mathrm{yr}^{-1}$, based on a sample of carbon stars complete within $\sim 600 \mathrm{pc}$ from the sun. This suggests that the stars in the sample presented here are going through the superwind phase of evolution (e.g. Vassiliadis \& Wood 1993) at the end of the AGB, and will soon eject the entire stellar mantle.

\subsection{CO isotopic ratios}

Using the envelope parameters derived from the ${ }^{12} \mathrm{CO}$ modelling, the abundance of ${ }^{13} \mathrm{CO}$ and $\mathrm{C}^{18} \mathrm{O}$ was estimated using the same radiative transfer code. The derived ${ }^{12} \mathrm{CO} /{ }^{13} \mathrm{CO}$-ratios are presented in Table 4 . The best fit ${ }^{13} \mathrm{CO}$ model obtained for AFGL 3068 is overlayed onto observations and presented in Fig. 3. The fits to the observed ${ }^{13} \mathrm{CO}$ emission are good in all cases with $\chi_{\text {red }}^{2} \lesssim 1$. The quality of the fits further strengthen the adopted physical models for the envelopes. The ${ }^{12} \mathrm{CO} /{ }^{13} \mathrm{CO}-$ ratio derived in this manner allows the assumption of optically thin emission adopted in Sect. 5 to be tested for those molecular species where the isotopomer containing ${ }^{13} \mathrm{C}$ has been detected. All the sample stars, except for IRAS 15194-5115 (to be discussed in Sect. 6.2), have inferred ${ }^{12} \mathrm{C} /{ }^{13} \mathrm{C}$-ratios in the range $\sim 20-50$. For optically bright carbon stars, i.e. generally lower mass-loss rate objects, values between $\sim 20-90$ are found (e.g., Schöier \& Olofsson 2000; Abia et al. 2001). The observed ${ }^{12} \mathrm{C} /{ }^{13} \mathrm{C}$-ratios are in agreement with evolutionary scenarios where the ${ }^{12} \mathrm{C} /{ }^{13} \mathrm{C}$-ratio is thought to increase from an initial low value of $\sim 10-25$, that depends on stellar mass, as the star evolves along the AGB (Abia et al. 2001). The thermal pulses that an AGB star experiences will effectively dredge 

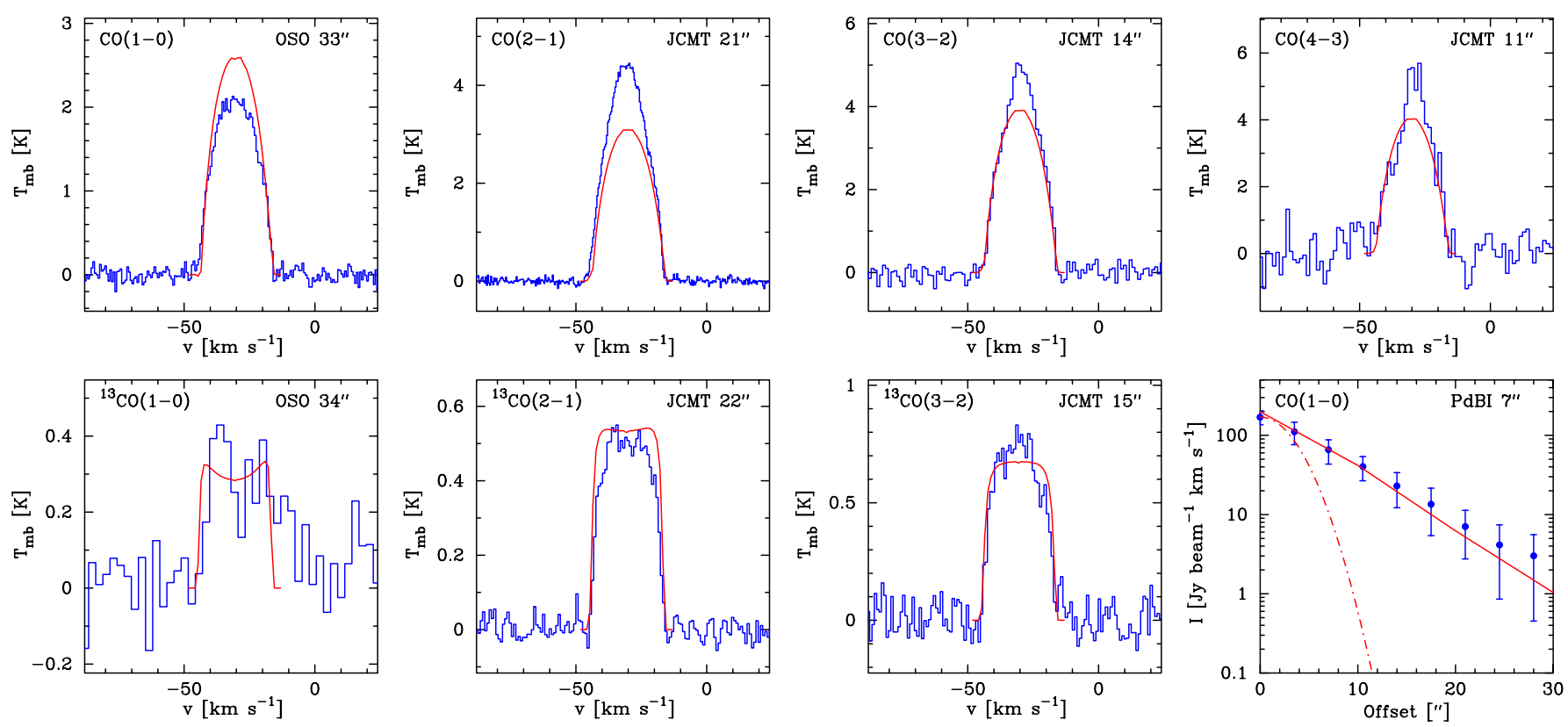

Fig. 3. Multi-transition CO millimetre-wave line emission observed towards AFGL 3068. The observed spectra (histograms) have been overlayed with the best fit model results (solid lines). Also shown, lower right panel, is the observed radial brightness distribution (boxes with error bars) overlayed by the results from the model (full line), with the circular beam used in the radiative transfer analysis (dot-dashed line). The transition, telescope used, and the corresponding beamsize, are indicated for each observation.

nucleosynthesized ${ }^{12} \mathrm{C}$ to the surface (Iben \& Renzini 1983) and, in addition to increasing the ${ }^{12} \mathrm{C} /{ }^{13} \mathrm{C}$-ratio, eventually turn it into a carbon star with $\mathrm{C} / \mathrm{O}>1$ in its atmosphere.

$\mathrm{C}^{18} \mathrm{O}$ emission was only detected towards IRC +10216 and a $\mathrm{C}^{16} \mathrm{O} / \mathrm{C}^{18} \mathrm{O}$-ratio of 1050 is derived, using four observational constraints (including three different transitions $J=1-0,2-1$, $3-2$ ) for $\mathrm{C}^{18} \mathrm{O}$. This value is in excellent agreement with the ${ }^{16} \mathrm{O} /{ }^{18} \mathrm{O}$-ratio of 1260 obtained by Kahane et al. (1992), using a combination of optically thin emission lines. In comparison, the value of this ratio in the solar neighbourhood is around 500 . The ${ }^{16} \mathrm{O} /{ }^{18} \mathrm{O}$-ratio and in particular the ${ }^{17} \mathrm{O} /{ }^{18} \mathrm{O}$-ratio, which is not measured here, can be used as tracers of nucleosynthesis. Like the ${ }^{12} \mathrm{C} /{ }^{13} \mathrm{C}$-ratio, these ratios are thought to increase as the star evolves along the AGB. Kahane et al. (1992) indeed find support for this scenario in a small sample of carbon-rich evolved stars.

\section{Abundance calculations}

A full radiative transfer analysis of the wealth of molecular data presented here is beyond the scope of this paper. A detailed treatment of the excitation would have to include the effects of dust emission and absorption in addition to accurate rates for collisional excitation of the molecules. Instead, abundances have been calculated for species with emission that is expected to be optically thin. In the case that the emission is optically thick, the calculated abundances using this assumption will be lower limits.

The calculation of isotope ratios of various species (as shown in Table 8) shows in general that many lines are optically thick (i.e., the ratio derived from observations is lower than the ${ }^{12} \mathrm{CO} /{ }^{13} \mathrm{CO}$ abundance ratio derived from the radiative transfer analysis). Where this is the case, the abundance of the main isotope (viz., $\mathrm{CN}, \mathrm{CS}$ and $\mathrm{HC}_{3} \mathrm{~N}$ ) has been calculated by scaling the abundance of the less abundant isotope by the ${ }^{12} \mathrm{CO} /{ }^{13} \mathrm{CO}-$ ratio. This is indicated by the bold-faced type in Table 7 . The abundance of $\mathrm{HCN}$ is not calculated using Eq. (3) since the line is certainly optically thick, but in all cases only by scaling the abundance of $\mathrm{H}^{13} \mathrm{CN}$ by the calculated ${ }^{12} \mathrm{CO} /{ }^{13} \mathrm{CO}$-ratio.

\subsection{Radiative transfer}

In the calculation of molecular abundances the circumstellar envelope is assumed to have been formed by a constant mass-loss rate and to expand with a constant velocity, such that the total density distribution follows an $r^{-2}$ law. It is further assumed that the fractional abundance of a species is constant in the radial range $r_{\mathrm{i}}$ to $r_{\mathrm{e}}$ and zero outside it. The excitation temperature was assumed to be constant throughout the emitting region. With these assumptions Olofsson et al. (1990) showed that for a given molecular transition,

$$
\begin{aligned}
\int T_{\mathrm{mb}} \mathrm{d} v= & \frac{c^{2}}{2 k v^{2}}\left[B_{v}\left(T_{\mathrm{ex}}\right)-B_{v}\left(T_{\mathrm{bg}}\right)\right] \\
& \times \frac{g_{\mathrm{u}} A_{\mathrm{ul}}}{8 \pi} \frac{c^{3}}{\nu^{3}} \frac{\left(1-\mathrm{e}^{-h v / k T_{\mathrm{ex}}}\right)}{Z} \mathrm{e}^{-E_{1} / k T_{\mathrm{ex}}} \\
& \times \frac{f_{\mathrm{X}} \dot{M}_{\mathrm{H}_{2}}}{v_{\mathrm{exp}} m_{\mathrm{H}_{2}} B D} \int_{x_{\mathrm{i}}}^{x_{\mathrm{e}}} \mathrm{e}^{-4 \ln (2) x^{2}} \mathrm{~d} x,
\end{aligned}
$$

where $c$ is the speed of light, $h$ the Planck constant, $k$ the Boltzmann constant, $B_{v}$ the Planck function, $T_{\mathrm{ex}}$ the excitation temperature, $T_{\mathrm{bg}}$ is taken to be the blackbody temperature of the cosmic background radiation at $2.7 \mathrm{~K}, m_{\mathrm{H}_{2}}$ is the mass of an $\mathrm{H}_{2}$ molecule, $v_{\exp }$ the expansion velocity of the circumstellar envelope, $B$ the $F W H M$ of the telescope beam, $D$ the assumed distance, $\dot{M}_{\mathrm{H}_{2}}$ the mass-loss rate, $Z$ the partition function, $v$ the frequency of the line, $g_{\mathrm{u}}$ the statistical weight of the upper level, $A_{\mathrm{ul}}$ the Einstein coefficient for the transition, $E_{1}$ the energy of 


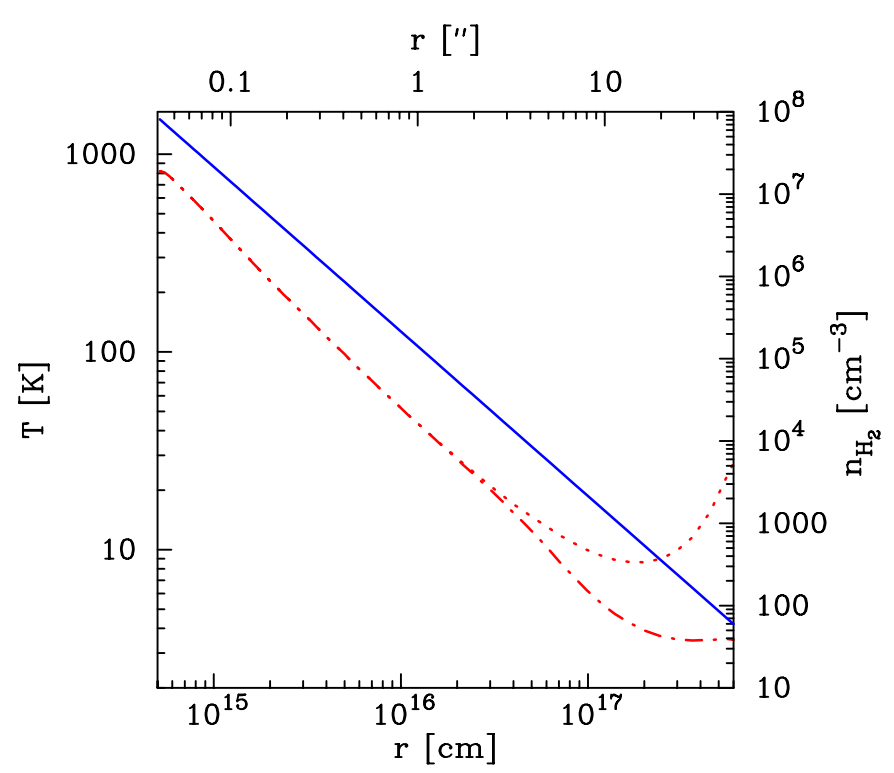

Fig. 4. The density (solid line) and kinetic temperature (dotted line) structures obtained from the CO excitation analysis for AFGL 3068. Also shown is the excitation temperature of the $\operatorname{CO}(J=2-1)$ line (dash-dotted line).

the lower transition level, and $x_{\mathrm{i}, \mathrm{e}}=r_{\mathrm{i}, \mathrm{e}} / B D$. The integral over $x$ takes care of the beam filling. However, if $r \ll B D$, the integral can be simplified to $\left(r_{\mathrm{e}}-r_{\mathrm{i}}\right) / B D$. This is the case in the furthest sources, IRAS 07454-7112, CIT 6 and IRAS 150824808. However, for the $\mathrm{CN}$ emission from these sources, which tends to be very extended, and for the remaining four sources, the full integral is calculated.

\subsection{The excitation temperature}

Of importance in the radiative transfer analysis is the excitation (rotational) temperature assumed for the molecular emission. In Fig. 4 the kinetic temperature and density structures for AFGL 3068 are shown. In the region where most of the molecular emission observed is thought to emanate from, $\sim 10^{16}-10^{17} \mathrm{~cm}$, the kinetic temperature ranges from $\sim 30-10 \mathrm{~K}$. Thus, if the emission were to be fully thermalized, then excitation temperatures would be expected to lie within this range. However, in addition to the low temperatures, the relatively low densities in this region, $\sim 10^{2}-10^{5} \mathrm{~cm}^{-3}$, makes the $\mathrm{CO}$ emission partly sub-thermally excited as illustrated in Fig. 4. Thus, sub-thermal emission is to be expected for all molecular species and the excitation temperature will depend on the species (and transition) observed.

When two or more transitions of the same molecule are observed, it is possible to make an estimate of the rotation temperature $\left(T_{\text {rot }}\right)$ of that molecule using Eq. (3). Assuming a molecular species to be excited over the same radial range, and according to a single temperature, the rotational temperature can be estimated. The results are shown in Table 5. It is clear that the rotational temperatures vary from source to source and between molecular species in the range $\sim 3-30 \mathrm{~K}$, as to be expected. The average excitation temperature is $8.7 \mathrm{~K}$ (averaged over the individual excitation temperatures for all sources, rather than molecular species). A generic value of $10 \mathrm{~K}$ was assumed for all the abundances estimated.

\subsection{The partition function}

All molecules are assumed to be linear, rigid rotators, except for $\mathrm{SiC}_{2}$ and $\mathrm{C}_{3} \mathrm{H}_{2}$ which are asymmetric tops and $\mathrm{CH}_{3} \mathrm{CN}$, which is a prolate symmetric top. Einstein A-coefficients (where available) and energy levels are taken from Chandra \& Rashmi (1998) for $\mathrm{SiC}_{2}$, from Vrtilek et al. (1987) for $\mathrm{C}_{3} \mathrm{H}_{2}$, and from Boucher et al. (1980) for $\mathrm{CH}_{3} \mathrm{CN}$.

The partition function, $Z$, is calculated assuming that no molecules have a hyperfine structure, i.e. as having simple rotational energy diagrams. Hence the integrated intensities which are summed over all hyperfine components are used. For $\mathrm{C}_{3} \mathrm{H}_{2}$, $\mathrm{CH}_{3} \mathrm{CN}$ and $\mathrm{SiC}_{2}$ we use the approximate expression for an asymmetric rotor (Townes \& Schawlow 1975) multiplied by 2 for $\mathrm{C}_{3} \mathrm{H}_{2}$, by 4 for $\mathrm{CH}_{3} \mathrm{CN}$ (to account for spin statistics) and by $1 / 2$ for $\mathrm{SiC}_{2}$ (since half of the energy levels are missing because of spin statistics). It is assumed that all levels are populated according to $T_{\mathrm{ex}}$.

\subsection{Sizes of emission regions}

\subsubsection{Photodissociation radii}

The chemical richness observed towards carbon stars can be qualitatively understood in terms of a photodissociation model (see the review by Glassgold 1996). Carbon-bearing molecules like $\mathrm{CO}, \mathrm{C}_{2} \mathrm{H}_{2}, \mathrm{HCN}$ and $\mathrm{CS}$, in addition to Sibearing molecules like $\mathrm{SiS}$ and $\mathrm{SiO}$, are all thought to be of photospheric origin where they are formed in conditions close to LTE. The photodissociation of these so-called parent species produces various radicals and ions that, in turn, drive a complex chemistry through ion-molecule and neutral-neutral reactions. For example, the radicals $\mathrm{C}_{2} \mathrm{H}$ and $\mathrm{CN}$ are the photodissociation products of $\mathrm{C}_{2} \mathrm{H}_{2}$ and $\mathrm{HCN}$, respectively. In addition, the formation of dust in the CSE will affect the abundances of some of the species, in particular $\mathrm{SiO}$ and $\mathrm{SiS}$, which condense onto dust grains. All other species observed in the sample are thought to be products of the circumstellar chemistry.

To calculate the radial extent of the molecules HCN, $\mathrm{CN}, \mathrm{C}_{2} \mathrm{H}$ and $\mathrm{CS}$ the photodissociation model of Huggins \& Glassgold (1982) is adopted. The photodissociation radius of a parent species $\left(f_{\mathrm{X}}\right.$, viz., $\left.\mathrm{HCN}, \mathrm{C}_{2} \mathrm{H}_{2}, \mathrm{CS}\right)$ is determined by

$\frac{\mathrm{d} f_{\mathrm{X}}}{\mathrm{d} r}=-\frac{G_{0, \mathrm{X}}}{v_{\exp }} \mathrm{e}^{-d_{\mathrm{X}} / r} f_{\mathrm{X}}$

and of a daughter $\left(f_{\mathrm{Xd}}\right.$, viz., $\left.\mathrm{C}_{2} \mathrm{H}\right)$ by

$\frac{\mathrm{d} f_{\mathrm{Xd}}}{\mathrm{d} r}=\frac{G_{0, \mathrm{X}}}{v_{\exp }} \mathrm{e}^{-d_{\mathrm{X}} / r} f_{\mathrm{X}}-\frac{G_{0, \mathrm{Xd}}}{v_{\exp }} \mathrm{e}^{-d_{\mathrm{Xd}} / r} f_{\mathrm{Xd}}$

$G_{0}$ is the unshielded photodissociation rate and $d_{\mathrm{X}}$ is the dust shielding distance, given by:

$d_{\mathrm{X}}=1.4 \frac{3 Q_{\mathrm{X}}}{4 a_{\mathrm{d}} \rho_{\mathrm{d}}} \frac{\dot{M}_{\mathrm{d}}}{4 \pi v_{\mathrm{d}}}$ 
Table 5. Rotation temperatures.

\begin{tabular}{lcccccc}
\hline \hline Source & ${ }^{13} \mathrm{CO}$ & $\mathrm{CN}$ & $\mathrm{CS}$ & $\mathrm{C}_{3} \mathrm{~N}$ & $\mathrm{C}_{4} \mathrm{H}$ & $\mathrm{HCN}$ \\
& $2-1 / 1-0$ & $2-1 / 1-0$ & $5-4 / 2-1$ & $11-10 / 10-9$ & $10-9 / 9-8$ & $3-2 / 2-1$ \\
\hline IRAS 07454-7112 & 7.0 & 5.2 & 11.3 & - & - & 5.7 \\
IRC+10216 (S) & 6.3 & 3.9 & 13.7 & 17.9 & 5.3 & 7.8 \\
IRAS 15082-4808 & 6.5 & 3.6 & 8.3 & - & - & 5.6 \\
IRAS 15194-5115 & 5.6 & 4.0 & 9.7 & - & - & 9.7 \\
Average & 6.4 & 4.2 & 10.8 & 17.9 & 5.3 & 7.2 \\
\hline \multicolumn{1}{r}{} & $\mathrm{H}^{(13)} \mathrm{C}_{3} \mathrm{~N}$ & $\mathrm{HC}_{3} \mathrm{~N}$ & $\mathrm{HC}_{5} \mathrm{~N}$ & $\mathrm{SiC}_{2}$ & $\mathrm{SiS}$ & \\
\hline Source & $12-11 / 10-9$ & $12-11 / 10-9$ & $34-33 / 32-31$ & $5-4 / 4-3$ & $6-5 / 5-4$ & Average \\
\hline IRAS 07454-7112 & 8.5 & 15.2 & - & - & 5.7 & 8.4 \\
IRC+10216 (S) & 17.8 & 10.7 & 10.0 & 11.6 & 5.8 & 10.1 \\
IRC+10216 (O) & - & - & - & - & 11.8 & 11.8 \\
IRAS 15082-4808 & - & 8.9 & - & 37.1 & 5.0 & 10.7 \\
IRAS 15194-5115 & 7.2 & 11.3 & - & - & 6.1 & 7.7 \\
IRC+40540 & - & - & - & - & 3.5 & 3.5 \\
Average & 11.2 & 11.5 & 10.0 & 24.4 & 6.3 & $\mathbf{8 . 7}$ \\
\hline
\end{tabular}

where $Q$ is the dust absorption efficiency, $\dot{M}_{\mathrm{d}}$ the dust massloss rate, and $v_{\mathrm{d}}$ the expansion velocity of the dust grains (Jura $\&$ Morris 1981). Since different molecules are generally dissociated at different wavelengths the adopted value of $Q$ will depend on the species under study. However, the wavelength dependence of $Q$ in the region of interest, $\sim 1000-3000 \AA$, is weak (e.g., Suh 2000) and a generic value of 1.0 for most species of interest here is adopted. However, the shielding distance of $\mathrm{CN}$ is taken to be a factor of 1.2 greater than that used for $\mathrm{HCN}$ (Truong-Bach et al. 1987). Introducing the $h$-parameter defined in Eq. (2) the dust shielding distance may now be expressed as

$d=5.2710^{22} \frac{h \dot{M}}{v_{\mathrm{d}}} \quad \mathrm{cm}$,

where the $\mathrm{H}_{2}$ mass-loss rate $\dot{M}$ is given in $M_{\odot} \mathrm{yr}^{-1}$ and $v_{\mathrm{d}}$ in $\mathrm{km} \mathrm{s}^{-1}$. A gas-to-dust mass ratio of 0.01 was assumed.

There will generally be a drift velocity between the dust and the gas (e.g., Schöier \& Olofsson 2001)

$v_{\mathrm{d}}-v_{\exp }=\sqrt{\frac{L v_{\exp }\langle Q\rangle}{\dot{M} c}}$,

where $L$ is the bolometric luminosity of the star, $c$ the speed of light, and $\langle Q\rangle$ is the flux-averaged momentum transfer efficiency. The drift velocity also enters in the expression of the heating caused by dust-gas collisions. In the self-consistent treatment of the energy balance in the circumstellar envelope a value of 0.03 was assumed for $\langle Q\rangle$, which is also retained here. Photodissocation rates are taken from van Dishoeck (1988), with the assumption that $\mathrm{SiS}$ has the same photodissociation rate as CS (confirmed to within a factor 2 by the UMIST Ratefile $^{2}$ ). The calculated shielding distances and rate coefficients are shown in Tables 4 and 6, respectively.

The photodissociation radii of species formed in the photosphere were chosen to be the e-folding radii of the initial

\footnotetext{
${ }^{2}$ http://www.rate99.co.uk
}

Table 6. Photodissociation rates.

\begin{tabular}{ll}
\hline \hline Molecule & $\begin{array}{c}G_{0} \\
{\left[\mathrm{~s}^{-1}\right]}\end{array}$ \\
\hline $\mathrm{HCN}$ & $1.1 \times 10^{-9}$ \\
$\mathrm{CN}$ & $2.5 \times 10^{-10}$ \\
$\mathrm{C}_{2} \mathrm{H}_{2}$ & $2.1 \times 10^{-9}$ \\
$\mathrm{C}_{2} \mathrm{H}$ & $3.4 \times 10^{-10}$ \\
$\mathrm{SiS}$ & $6.3 \times 10^{-10}$ \\
$\mathrm{CS}$ & $6.3 \times 10^{-10}$ \\
$\mathrm{SiO}$ & $6.3 \times 10^{-10}$ \\
\hline
\end{tabular}

abundances, i.e. $f_{\mathrm{X}}\left(r_{\mathrm{e}}\right)=f_{\mathrm{X}}\left(R_{\star}\right) / \mathrm{e}$, where $R_{\star}$ is the stellar radius. For the photodissociation products inner and outer radii are chosen to be where the abundance has dropped to $1 / \mathrm{e}$ of its peak value. The envelope sizes calculated by the simple photodissociation model agree relatively well with observed values in IRC +10216 and other objects, except in the case of SiS (see next paragraph). Lindqvist et al. (2000) used a detailed radiative transfer method and observed brightness distributions to derive envelope sizes of $\mathrm{HCN}$ and CN. For IRC +10216 and IRC +40540 they found envelope sizes of $\sim 4 \times 10^{16} \mathrm{~cm}$ for $\mathrm{HCN}$ and $\sim 5 \times 10^{16} \mathrm{~cm}$ for $\mathrm{CN}$, in excellent agreement with the values derived here from the photodissociation model. In the case of CIT 6 the observed HCN envelope size is larger than that calculated by a factor of 2 . Envelope sizes and derived abundances are given in Table 7.

$\mathrm{SiO}$ and $\mathrm{SiS}$ are parent species with a radial extent depending on photodissociation. The calculation of the SiS photodissociation radius in IRC +10216 , however, is not consistent with the radius observed by Bieging \& Tafalla (1993). Therefore the observed radius for $\mathrm{SiS}$ in $\mathrm{IRC}+10216$ was used in the abundance calculations, and its radius was scaled for the other objects in the sample with the factor $\left(\dot{M} / v_{\exp }\right)_{\star} /\left(\dot{M} / v_{\exp }\right)_{10216}$ in the same way as described in Sect. 5.4.2. The outer radius calculated via the photodissociation model is actually slightly larger than that observed. This gives credence to the idea that 
$\mathrm{SiS}$ freezes out onto dust grains. $\mathrm{SiO}$ is treated in the same way as SiS since it too is likely to freeze out onto grains. Hence the same envelope size as SiS was adopted for this species.

\subsubsection{Inner and outer radii for species of circumstellar chemistry origin}

For those molecules that have their origin in a circumstellar chemistry a different approach is used. The sizes of the emission regions of $\mathrm{HNC}$ and $\mathrm{HC}_{3} \mathrm{~N}$ have been determined interferometrically for IRC +10216 using observations by Bieging \& Rieu (1988), and of $\mathrm{SiC}_{2}$ by Lucas et al. (1995). Inner and outer radii for $\mathrm{HNC}, \mathrm{HC}_{3} \mathrm{~N}$ and $\mathrm{SiC}_{2}$ are taken from observations of IRC +10216 , and scaled by a factor $\left(\dot{M} / v_{\text {exp }}\right)_{\star} /\left(\dot{M} / v_{\text {exp }}\right)_{10216}$ for the remaining six sample carbon stars (since molecular distributions have not been mapped in all the sample stars; see Table 4 for values of this ratio). This factor is proportional to the density ratio at a given radius, and also (to the first order) to the ratio of the envelope shielding distances. Chemical modelling shows that the species $\mathrm{C}_{3} \mathrm{H}, \mathrm{C}_{4} \mathrm{H}, \mathrm{C}_{3} \mathrm{H}_{2}, \mathrm{C}_{3} \mathrm{~N}, \mathrm{CH}_{3} \mathrm{CN}$, $\mathrm{HC}_{5} \mathrm{~N}, \mathrm{SO}, \mathrm{C}_{2} \mathrm{~S}$ and $\mathrm{C}_{3} \mathrm{~S}$ are formed via chemical reactions in the outer envelope (e.g., Millar \& Herbst 1994). Furthermore, it shows that the species $\mathrm{C}_{3} \mathrm{H}, \mathrm{C}_{4} \mathrm{H}, \mathrm{C}_{3} \mathrm{H}_{2}$ and $\mathrm{C}_{3} \mathrm{~N}$ have a similar radial distribution to $\mathrm{HNC}$, and that the species $\mathrm{CH}_{3} \mathrm{CN}$ and $\mathrm{HC}_{5} \mathrm{~N}$ are similarly distributed to $\mathrm{HC}_{3} \mathrm{~N}$. Hence corresponding radii for these species are assumed in the calculations. SO is assumed to have a similar distribution to $\mathrm{CN}$ (cf., Nejad \& Millar 1988), and $C_{2} S$ and $C_{3} S$ are assumed to follow the CS distribution (cf., Millar et al. 2001), although these latter two species are not parent molecules (and hence are given an inner radius equal to that of $\mathrm{HC}_{3} \mathrm{~N}$ ). All isotopes (i.e. species involving ${ }^{13} \mathrm{C}$ and ${ }^{34} \mathrm{~S}$ ) are further assumed to have the same distributions as the main isotope.

\subsection{Molecular abundances}

\subsubsection{Uncertainties in the abundance estimates}

The assumption of optically thin emission has a systematic effect on the derived abundances, in that the true abundance will be higher if there are opacity effects. As can be seen from Table 8 , there can be a factor of $2-3$ in error from this assumption.

The accuracy of the calculated abundances is dependent on various assumptions. Due to the intrinsic difficulties in estimating distances to the objects included in the sample, the typical uncertainty in the adopted distance is a factor of $\sim 2$. This influences the mass-loss rates derived in the radiative transfer modelling such that the adopted mass-loss rate will scale as $D^{1-2}$. The calculation of the limiting radii of a certain molecular distribution in the envelope is also affected by inaccuracies in the distance estimate. Where radii have been calculated by scaling observed radii in IRC +10216 , there comes an error which scales as $D$. The results from the photodissociation model will have a lesser dependence, with $D$ coming into the expression for the shielding distance, via $\dot{M}$. Hence the abundance, which is trivially derived from Eq. (3), will vary as approximately $D^{-1-0}$, giving a factor of 2, possibly, in error.
In the present analysis an excitation temperature of $10 \mathrm{~K}$ was assumed for all transitions. The error in abundance estimate due to this assumption will depend on the excitation temperature of a particular transition and the assumption of LTE, and is estimated to be not more than a factor of $\sim 2$.

Overall, it seems like an error of a factor of 5 is reasonable to expect in the abundances presented here, although it should be borne in mind that this could possibly rise to an order of magnitude. In the comparison of abundance ratios in Table 9, any difference less than a factor of 5 is treated as insignificant with respect to error margins.

\subsubsection{Comparison with previously published abundances}

In comparison with Nyman et al. (1993), derived abundance ratios (Table 9) have increased in favour of IRAS 151945115 by up to a factor of approximately 4 . Individual abundances generally show a factor $\sim 2$ increase for those in IRAS 15914-5115, and a factor $\sim 2$ decrease for IRC +10216 , over Nyman et al. (1993). In Nyman et al. (1993), the distances adopted for IRC +10216 and IRAS 15194-5115 were approximately twice as large as those here; however this has little effect on calculated abundances since the distance ratio is more or less unchanged. However, the calculated mass-loss rate of IRAS $15194-5115$ in the previous paper was larger than that of IRC +10216 by a factor of 2.5 , and here the recalculated massloss rates are the same for these two objects. This would tend to increase the abundance ratios quoted by a similar factor. The photodissociation radius depends on the mass-loss rate and the dust parameters through the dust shielding distance. Compared to Nyman et al. (1993) this paper uses different mass-loss rates, a different gas-to-dust ratio (0.01 compared to 0.005$)$, and the dust parameters are also slightly different due to the determination of the $h$-factor in the radiative transfer analysis. The scale factor that determines the inner and outer radii of the species with a origin in circumstellar chemistry depends on the ratio of the mass-loss rates. Thus the difference in relative mass loss rates explains the factor of 4 difference in relative abundances between IRAS 15914-5115 and IRC +10216 derived in this paper compared to those derived in Nyman et al. (1993).

As discussed earlier in this paper the outer radii of $\mathrm{SiS}$ and $\mathrm{SiO}$ are not determined through the photodissociation radius but scaled from their observed radius in IRC +10216 . In this way the calculated abundances of $\mathrm{SiO}$ and $\mathrm{SiS}$ have increased in by a factor of 4-5 for IRAS 15194-5115 compared to Nyman et al. (1993). For IRC +10216 these species have the same abundance as calculated previously, and they are in reasonable agreement with those reported elsewhere in the literature. Bujarrabal et al. (1994) give $f(\mathrm{SiO})=5.6 \times 10^{-7}$ and $f(\mathrm{SiS})=3.9 \times 10^{-6}$, which are $\sim 4$ times greater, using an outer radius half that quoted in this paper and a distance of $200 \mathrm{pc}$. Better agreement is seen for the other species which Bujarrabal et al. (1994) detect, with the exception of HNC, which is a factor $\sim 6$ in disagreement. Cernicharo et al. (2000) also calculate abundances in IRC +10216 , and are within a factor 5 of those here. 
Table 7. Abundances and sizes of emission regions ${ }^{a}$.

\begin{tabular}{|c|c|c|c|c|c|c|c|c|c|c|c|c|}
\hline \multirow[t]{2}{*}{ Molecule } & \multicolumn{3}{|c|}{ IRC $+10216($ SEST $)$} & \multicolumn{3}{|c|}{ IRC $+10216(\mathrm{OSO})$} & \multicolumn{3}{|c|}{ IRAS $15194-5115$} & \multicolumn{3}{|c|}{ IRAS $15082-4808$} \\
\hline & $\begin{array}{c}r_{\mathrm{i}} \\
{[\mathrm{cm}]}\end{array}$ & $\begin{array}{c}r_{\mathrm{e}} \\
{[\mathrm{cm}]}\end{array}$ & $\begin{array}{c}f_{\mathrm{X}} \\
{[\mathrm{X}] /\left[\mathrm{H}_{2}\right]}\end{array}$ & $\begin{array}{c}r_{\mathrm{i}} \\
{[\mathrm{cm}]}\end{array}$ & $\begin{array}{c}r_{\mathrm{e}} \\
{[\mathrm{cm}]}\end{array}$ & $\begin{array}{c}f_{\mathrm{X}} \\
{[\mathrm{X}] /\left[\mathrm{H}_{2}\right]}\end{array}$ & $\begin{array}{c}r_{\mathrm{i}} \\
{[\mathrm{cm}]}\end{array}$ & $\begin{array}{c}r_{\mathrm{e}} \\
{[\mathrm{cm}]}\end{array}$ & $\begin{array}{c}f_{\mathrm{X}} \\
{[\mathrm{X}] /\left[\mathrm{H}_{2}\right]}\end{array}$ & $\begin{array}{c}r_{\mathrm{i}} \\
{[\mathrm{cm}]}\end{array}$ & $\begin{array}{c}r_{\mathrm{e}} \\
{[\mathrm{cm}]}\end{array}$ & $\begin{array}{c}f_{\mathrm{X}} \\
{[\mathrm{X}] /\left[\mathrm{H}_{2}\right]}\end{array}$ \\
\hline $\mathrm{CN}(1-0)$ & $2.6(16)$ & $7.1(16)$ & $3.4(-06)$ & $2.6(16)$ & $7.1(16)$ & $2.1(-06)$ & $3.5(16)$ & $9.6(16)$ & $2.1(-06)$ & $2.0(16)$ & $6.2(16)$ & $3.2(-06)$ \\
\hline${ }^{13} \mathrm{CN}(1-0)$ & $2.6(16)$ & $7.1(16)$ & $7.6(-08)$ & $2.6(16)$ & $7.1(16)$ & $4.6(-08)$ & $3.5(16)$ & $9.6(16)$ & $3.0(-07)$ & $2.0(16)$ & $6.2(16)$ & $<1.3(-08)$ \\
\hline $\mathrm{CN}(2-1)$ & $2.6(16)$ & $7.1(16)$ & $9.6(-07)$ & $2.6(16)$ & $7.1(16)$ & - & $3.5(16)$ & $9.6(16)$ & $3.5(-07)$ & $2.0(16)$ & $6.2(16)$ & $3.8(-07)$ \\
\hline $\operatorname{CS}(2-1)$ & & $4.0(16)$ & $9.9(-07)$ & & $4.0(16)$ & $4.6(-07)$ & & $5.5(16)$ & $2.3(-06)$ & & $3.3(16)$ & $2.2(-06)$ \\
\hline${ }^{13} \operatorname{CS}(2-1)$ & & $4.0(16)$ & $2.2(-08)$ & & $4.0(16)$ & - & & $5.5(16)$ & $5.0(-07)$ & & $3.3(16)$ & $<3.1(-08)$ \\
\hline$C^{34} S(2-1)$ & & $4.0(16)$ & $3.7(-08)$ & & $4.0(16)$ & - & & $5.5(16)$ & - & & $3.3(16)$ & - \\
\hline $\operatorname{CS}(5-4)$ & & $4.0(16)$ & $1.2(-06)$ & & $4.0(16)$ & - & & $5.5(16)$ & $1.6(-06)$ & & $3.3(16)$ & $9.0(-07)$ \\
\hline $\mathrm{C}_{2} \mathrm{~S}(6,7-5,6)$ & $1.2(16)$ & $4.0(16)$ & $<4.6(-09)$ & $1.2(16)$ & $4.0(16)$ & $<4.5(-09)$ & $8.0(15)$ & $5.5(16)$ & $<2.4(-08)$ & $7.4(15)$ & $3.3(16)$ & $<3.1(-08)$ \\
\hline $\mathrm{C}_{2} \mathrm{~S}(8,9-7,8)$ & $1.2(16)$ & $4.0(16)$ & $<4.1(-09)$ & $1.2(16)$ & $4.0(16)$ & $<9.5(-09)$ & $8.0(15)$ & $5.5(16)$ & $<3.5(-08)$ & $7.4(15)$ & $3.3(16)$ & $<4.8(-08)$ \\
\hline$C_{3} S(15-14)$ & $1.2(16)$ & $4.0(16)$ & $<1.2(-08)$ & $1.2(16)$ & $4.0(16)$ & $<1.5(-08)$ & $8.0(15)$ & $5.5(16)$ & $<7.2(-08)$ & $7.4(15)$ & $3.3(16)$ & $<1.3(-06)$ \\
\hline $\mathrm{SiO}(2-1)$ & & $2.0(16)$ & $1.3(-07)$ & & $2.0(16)$ & $9.6(-08)$ & & $1.3(16)$ & $1.7(-06)$ & & $3.3(16)$ & $7.2(-07)$ \\
\hline $\operatorname{SiS}(5-4)$ & & $2.0(16)$ & $1.2(-06)$ & & $2.0(16)$ & $8.1(-07)$ & & $1.3(16)$ & $4.9(-06)$ & & $3.3(16)$ & $3.3(-06)$ \\
\hline $\operatorname{SiS}(6-5)$ & & $2.0(16)$ & $9.0(-07)$ & & $2.0(16)$ & $9.0(-07)$ & & $1.3(16)$ & $4.2(-06)$ & & $3.3(16)$ & $2.0(-06)$ \\
\hline $\mathrm{SO}(3-2)$ & $2.6(16)$ & $7.1(16)$ & $<3.5(-09)$ & $2.6(16)$ & $7.1(16)$ & - & $3.5(16)$ & $9.6(16)$ & - & $2.0(16)$ & $6.2(16)$ & - \\
\hline $\mathrm{HCN}(1-0)$ & & $3.4(16)$ & $1.4(-05)$ & & $3.4(16)$ & $1.1(-05)$ & & $4.6(16)$ & $1.2(-05)$ & & $2.7(16)$ & $1.0(-05)$ \\
\hline $\mathrm{H}^{13} \mathrm{CN}(1-0)$ & & $3.4(16)$ & $3.1(-07)$ & & $3.4(16)$ & $2.5(-07)$ & & 4.6(16) & $2.0(-06)$ & & $2.7(16)$ & $2.9(-07)$ \\
\hline $\mathrm{HNC}(1-0)$ & $2.4(16)$ & $8.4(16)$ & $7.2(-08)$ & $2.4(16)$ & $8.4(16)$ & $3.8(-08)$ & $1.6(16)$ & $5.6(16)$ & $3.3(-07)$ & $1.5(16)$ & $5.2(16)$ & $1.6(-07)$ \\
\hline $\mathrm{HN}^{13} \mathrm{C}(1-0)$ & $2.4(16)$ & $8.4(16)$ & $<1.9(-09)$ & $2.4(16)$ & $8.4(16)$ & - & $1.6(16)$ & $5.6(16)$ & $1.1(-07)$ & $1.5(16)$ & $5.2(16)$ & $<1.2(-08)$ \\
\hline $\mathrm{SiC}_{2}(4,04-3,03)$ & $2.4(16)$ & $6.0(16)$ & $1.6(-07)$ & $2.4(16)$ & $6.0(16)$ & - & $1.6(16)$ & $4.0(16)$ & - & $1.5(16)$ & $3.7(16)$ & $3.3(-07)$ \\
\hline $\mathrm{SiC}_{2}(4,22-3,21)$ & $2.4(16)$ & $6.0(16)$ & $3.2(-07)$ & $2.4(16)$ & $6.0(16)$ & - & $1.6(16)$ & $4.0(16)$ & - & $1.5(16)$ & $3.7(16)$ & $<1.8(-07)$ \\
\hline $\mathrm{SiC}_{2}(5,05-4,04)$ & $2.4(16)$ & $6.0(16)$ & $1.7(-07)$ & $2.4(16)$ & $6.0(16)$ & $1.7(-07)$ & $1.6(16)$ & $4.0(16)$ & $1.2(-06)$ & $1.5(16)$ & $3.7(16)$ & $4.9(-07)$ \\
\hline $\mathrm{C}_{2} \mathrm{H}(1-0)$ & $2.3(16)$ & $5.6(16)$ & $2.8(-06)$ & $2.3(16)$ & $5.6(16)$ & $2.4(-06)$ & $1.6(16)$ & $4.8(16)$ & $1.5(-05)$ & $1.7(16)$ & $4.8(16)$ & $8.3(-06)$ \\
\hline $\mathrm{C}_{3} \mathrm{H}(9 / 2-7 / 2)$ & $2.4(16)$ & $8.4(16)$ & $5.5(-08)$ & $2.4(16)$ & $8.4(16)$ & $5.9(-08)$ & $1.6(16)$ & $5.6(16)$ & $9.2(-08)$ & $1.5(16)$ & $5.2(16)$ & $<1.7(-08)$ \\
\hline $\mathrm{C}_{3} \mathrm{~N}(9-8)$ & $2.4(16)$ & $8.4(16)$ & $3.0(-07)$ & $2.4(16)$ & $8.4(16)$ & - & $1.6(16)$ & $5.6(16)$ & $<4.9(-08)$ & $1.5(16)$ & $5.2(16)$ & $<1.0(-07)$ \\
\hline $\mathrm{C}_{3} \mathrm{~N}(11-10)$ & $2.4(16)$ & $8.4(16)$ & $5.9(-07)$ & $2.4(16)$ & $8.4(16)$ & $7.6(-07)$ & $1.6(16)$ & $5.6(16)$ & $9.2(-07)$ & $1.5(16)$ & $5.2(16)$ & $9.5(-07)$ \\
\hline $\mathrm{C}_{4} \mathrm{H}(9-8)$ & $2.4(16)$ & $8.4(16)$ & $3.7(-06)$ & $2.4(16)$ & $8.4(16)$ & - & $1.6(16)$ & $5.6(16)$ & - & $1.5(16)$ & $5.2(16)$ & $<6.6(-07)$ \\
\hline $\mathrm{C}_{4} \mathrm{H}(10-9)$ & $2.4(16)$ & $8.4(16)$ & $2.7(-06)$ & $2.4(16)$ & $8.4(16)$ & $3.2(-06)$ & $1.6(16)$ & $5.6(16)$ & $2.8(-05)$ & $1.5(16)$ & $5.2(16)$ & $6.9(-06)$ \\
\hline $\mathrm{C}_{3} \mathrm{H}_{2}(2,12-1,01)$ & $2.4(16)$ & $8.4(16)$ & $3.2(-08)$ & $2.4(16)$ & $8.4(16)$ & - & $1.6(16)$ & $5.6(16)$ & $6.0(-07)$ & $1.5(16)$ & $5.2(16)$ & $<9.3(-08)$ \\
\hline $\mathrm{HC}_{3} \mathrm{~N}(10-9)$ & $1.2(16)$ & $6.0(16)$ & $1.1(-06)$ & $1.2(16)$ & $6.0(16)$ & $4.4(-07)$ & $8.0(15)$ & $4.0(16)$ & $1.9(-06)$ & $7.4(15)$ & $3.7(16)$ & $2.3(-06)$ \\
\hline $\mathrm{HCC}^{13} \mathrm{CN}(10-9)$ & $1.2(16)$ & $6.0(16)$ & $2.4(-08)$ & $1.2(16)$ & $6.0(16)$ & $<2.8(-09)$ & $8.0(15)$ & $4.0(16)$ & $3.9(-07)$ & $7.4(15)$ & $3.7(16)$ & $<2.7(-08)$ \\
\hline $\mathrm{HC}^{13} \mathrm{CCN}(10-9)$ & $1.2(16)$ & $6.0(16)$ & $2.4(-08)$ & $1.2(16)$ & $6.0(16)$ & $<2.8(-09)$ & $8.0(15)$ & $4.0(16)$ & $3.9(-07)$ & $7.4(15)$ & $3.7(16)$ & $<2.7(-08)$ \\
\hline $\mathrm{HC}_{3} \mathrm{~N}(11-10)$ & $1.2(16)$ & $6.0(16)$ & $7.5(-07)$ & $1.2(16)$ & $6.0(16)$ & - & $8.0(15)$ & $4.0(16)$ & - & $7.4(15)$ & $3.7(16)$ & - \\
\hline $\mathrm{HC}_{3} \mathrm{~N}(12-11)$ & $1.2(16)$ & $6.0(16)$ & $1.9(-06)$ & $1.2(16)$ & $6.0(16)$ & - & $8.0(15)$ & $4.0(16)$ & $2.2(-06)$ & $7.4(15)$ & $3.7(16)$ & $2.1(-06)$ \\
\hline $\mathrm{HCC}^{13} \mathrm{CN}(12-11)$ & $1.2(16)$ & $6.0(16)$ & $4.1(-08)$ & $1.2(16)$ & $6.0(16)$ & $1.5(-08)$ & $8.0(15)$ & $4.0(16)$ & $2.8(-07)$ & $7.4(15)$ & $3.7(16)$ & $<2.0(-08)$ \\
\hline $\mathrm{HC}^{13} \mathrm{CCN}(12-11)$ & $1.2(16)$ & $6.0(16)$ & $4.1(-08)$ & $1.2(16)$ & $6.0(16)$ & $1.5(-08)$ & $8.0(15)$ & $4.0(16)$ & $2.8(-07)$ & $7.4(15)$ & $3.7(16)$ & $<2.0(-08)$ \\
\hline $\mathrm{CH}_{3} \mathrm{CN}(6(1)-5(1))$ & $1.2(16)$ & $6.0(16)$ & $1.2(-08)$ & $1.2(16)$ & $6.0(16)$ & $<2.0(-09)$ & $8.0(15)$ & $4.0(16)$ & $<2.5(-08)$ & $7.4(15)$ & $3.7(16)$ & $<4.3(-08)$ \\
\hline $\mathrm{CH}_{3} \mathrm{CN}(12(0)-11(0))$ & $1.2(16)$ & $6.0(16)$ & $<1.2(-07)$ & $1.2(16)$ & $6.0(16)$ & - & $8.0(15)$ & $4.0(16)$ & $<2.4(-06)$ & $7.4(15)$ & $3.7(16)$ & $<1.5(-06)$ \\
\hline $\mathrm{HC}_{5} \mathrm{~N}(32-31)$ & $1.2(16)$ & $6.0(16)$ & $3.9(-06)$ & $1.2(16)$ & $6.0(16)$ & - & $8.0(15)$ & $4.0(16)$ & $<4.5(-06)$ & $7.4(15)$ & $3.7(16)$ & $<3.8(-06)$ \\
\hline $\mathrm{HC}_{5} \mathrm{~N}(34-33)$ & $1.2(16)$ & $6.0(16)$ & $4.2(-06)$ & $1.2(16)$ & $6.0(16)$ & $<7.6(-07)$ & $8.0(15)$ & $4.0(16)$ & - & $7.4(15)$ & $3.7(16)$ & $<7.4(-06)$ \\
\hline $\mathrm{HC}_{5} \mathrm{~N}(35-34)$ & $1.2(16)$ & $6.0(16)$ & $1.3(-05)$ & $1.2(16)$ & $6.0(16)$ & - & $8.0(15)$ & $4.0(16)$ & - & $7.4(15)$ & $3.7(16)$ & $<1.1(-05)$ \\
\hline
\end{tabular}

${ }^{a}$ In this table $x(y)$ represents $x \times 10^{y}$.

${ }^{b}$ Bold face indicates an abundance calculated by scaling the abundance of the ${ }^{13} \mathrm{C}$ isotope of the same species by the modelled

${ }^{12} \mathrm{CO} /{ }^{13} \mathrm{CO}$-ratio (see Sect. 5.5).

Generally, it seems that IRC +10216 has lower abundances than IRAS 15194-5115, IRAS 15082-4808, IRAS 074547112 and CIT 6 . However, it is very similar, physically and chemically, to AFGL 3068 and IRC +40540 .

The three northern sources observed at OSO have also been studied in the literature, and abundances derived. The Bujarrabal et al. (1994) paper includes data relating to CIT 6, AFGL 3068 and IRC +40540. All abundances calculated by Bujarrabal et al. in these three sources are greater than those derived here. Distances and mass-loss rates are reasonably comparable between this paper and that. Generally, this means that the calculated abundances in this paper are lower by a factor of $\sim 2$ in CIT 6, a factor of $\sim 5$ in AFGL 3068, and a factor of $\sim 3$ in IRC +40540 compared to those derived in Bujarrabal et al. (1994).

\section{Discussion}

\subsection{Chemistry}

\subsubsection{Presence of shocks in the inner wind?}

When thermal equilibrium (TE) prevails the molecular content of a gas can be readily calculated from its elemental chemical composition. This is the case in the stellar photosphere and near the inner boundary of the envelope of an AGB-star, where the gas density and temperature are high. The variable nature 
Table 7. continued.

\begin{tabular}{|c|c|c|c|c|c|c|c|c|c|c|c|c|}
\hline \multirow[t]{2}{*}{ Molecule } & \multicolumn{3}{|c|}{ IRAS 07454-7112 } & \multicolumn{3}{|c|}{ CIT 6} & \multicolumn{3}{|c|}{ AFGL 3068} & \multicolumn{3}{|c|}{ IRC +40540} \\
\hline & $\begin{array}{c}r_{\mathrm{i}} \\
\mathrm{cm}\end{array}$ & $\begin{array}{l}r_{\mathrm{e}} \\
\mathrm{cm}\end{array}$ & $\begin{array}{c}f_{\mathrm{X}} \\
{[\mathrm{X}] /\left[\mathrm{H}_{2}\right]}\end{array}$ & $\begin{array}{l}r_{\mathrm{i}} \\
\mathrm{cm}\end{array}$ & $\begin{array}{l}r_{\mathrm{e}} \\
\mathrm{cm}\end{array}$ & $\begin{array}{c}f_{\mathrm{X}} \\
{[\mathrm{X}] /\left[\mathrm{H}_{2}\right]}\end{array}$ & $\begin{array}{l}r_{\mathrm{i}} \\
\mathrm{cm}\end{array}$ & $\begin{array}{l}r_{\mathrm{e}} \\
\mathrm{cm}\end{array}$ & $\begin{array}{c}f_{\mathrm{X}} \\
{[\mathrm{X}] /\left[\mathrm{H}_{2}\right]}\end{array}$ & $\begin{array}{l}r_{\mathrm{i}} \\
\mathrm{cm}\end{array}$ & $\begin{array}{l}r_{\mathrm{e}} \\
\mathrm{cm}\end{array}$ & $\begin{array}{c}f_{\mathrm{X}} \\
{[\mathrm{X}] /\left[\mathrm{H}_{2}\right]}\end{array}$ \\
\hline $\mathrm{CN}(1-0)$ & $1.3(16)$ & $4.1(16)$ & $5.3(-06)$ & $1.6(16)$ & $5.1(16)$ & $2.0(-05)$ & $3.2(16)$ & $8.3(16)$ & $4.6(-07)$ & $3.2(16)$ & $8.3(16)$ & $1.0(-06)$ \\
\hline${ }^{13} \mathrm{CN}(1-0)$ & $1.3(16)$ & $4.1(16)$ & $4.8(-07)$ & $1.6(16)$ & $5.1(16)$ & $5.8(-07)$ & $3.2(16)$ & $8.3(16)$ & $<2.6(-08)$ & $3.2(16)$ & $8.3(16)$ & $<9.9(-09)$ \\
\hline $\mathrm{CN}(2-1)$ & $1.3(16)$ & $4.1(16)$ & $1.6(-06)$ & $1.6(16)$ & $5.1(16)$ & - & $3.2(16)$ & $8.3(16)$ & - & $3.2(16)$ & $8.3(16)$ & - \\
\hline $\operatorname{CS}(2-1)$ & & $2.2(16)$ & $1.6(-06)$ & & $2.7(16)$ & $2.5(-06)$ & & $4.8(16)$ & $3.7(-07)$ & & $4.9(16)$ & $5.4(-07)$ \\
\hline${ }^{13} \mathrm{CS}(2-1)$ & & $2.2(16)$ & $<2.9(-08)$ & & $2.7(16)$ & - & & $4.8(16)$ & - & & $4.9(16)$ & - \\
\hline$C^{34} S(2-1)$ & & $2.2(16)$ & - & & $2.7(16)$ & - & & $4.8(16)$ & - & & $4.9(16)$ & - \\
\hline $\operatorname{CS}(5-4)$ & & $2.2(16)$ & $1.6(-06)$ & & $2.7(16)$ & - & & $4.8(16)$ & - & & $4.9(16)$ & - \\
\hline $\mathrm{C}_{2} \mathrm{~S}(6,7-5,6)$ & $5.6(15)$ & $2.2(16)$ & $<9.1(-08)$ & $4.3(15)$ & $2.7(16)$ & $<1.0(-07)$ & $1.6(16)$ & $4.8(16)$ & $<8.1(-08)$ & $1.6(16)$ & $4.9(16)$ & $<3.0(-08)$ \\
\hline $\mathrm{C}_{2} \mathrm{~S}(8,9-7,8)$ & $5.6(15)$ & $2.2(16)$ & $<1.1(-07)$ & $4.3(15)$ & $2.7(16)$ & $<2.0(-07)$ & $1.6(16)$ & $4.8(16)$ & $<8.0(-08)$ & $1.6(16)$ & $4.9(16)$ & $<5.6(-08)$ \\
\hline $\mathrm{C}_{3} \mathrm{~S}(15-14)$ & $5.6(15)$ & $2.2(16)$ & $<3.6(-07)$ & $4.3(15)$ & $2.7(16)$ & $<2.5(-07)$ & $1.6(16)$ & $4.8(16)$ & $<3.5(-07)$ & $1.6(16)$ & $4.9(16)$ & $<8.9(-08)$ \\
\hline $\mathrm{SiO}(2-1)$ & & $9.4(15)$ & $4.4(-07)$ & & $7.2(15)$ & $1.0(-06)$ & & $2.6(16)$ & $<4.7(-08)$ & & $2.6(16)$ & $5.6(-08)$ \\
\hline $\operatorname{SiS}(5-4)$ & & $9.4(15)$ & $4.8(-06)$ & & $7.2(15)$ & $1.0(-06)$ & & $2.6(16)$ & $3.3(-07)$ & & $2.6(16)$ & $1.4(-06)$ \\
\hline $\operatorname{SiS}(6-5)$ & & $9.4(15)$ & $3.4(-06)$ & & $7.2(15)$ & $5.2(-06)$ & & $2.6(16)$ & $1.0(-06)$ & & $2.6(16)$ & $4.9(-07)$ \\
\hline $\mathrm{HCN}(1-0)$ & & $1.8(16)$ & $7.8(-06)$ & & $2.2(16)$ & $1.1(-05)$ & & $4.2(16)$ & $6.3(-06)$ & & $4.2(16)$ & $6.5(-06)$ \\
\hline $\mathrm{H}^{13} \mathrm{CN}(1-0)$ & & $1.8(16)$ & $5.6(-07)$ & & $2.2(16)$ & $3.0(-07)$ & & $4.2(16)$ & $2.1(-07)$ & & $4.2(16)$ & $1.3(-07)$ \\
\hline $\mathrm{HNC}(1-0)$ & $1.1(16)$ & $4.0(16)$ & $1.0(-07)$ & $8.6(15)$ & $3.0(16)$ & $2.3(-07)$ & $3.1(16)$ & $1.1(17)$ & $3.0(-08)$ & $3.1(16)$ & $1.1(17)$ & $2.2(-08)$ \\
\hline $\mathrm{HN}^{13} \mathrm{C}(1-0)$ & $1.1(16)$ & $4.0(16)$ & $<1.8(-08)$ & $8.6(15)$ & $3.0(16)$ & $<2.1(-08)$ & $3.1(16)$ & $1.1(17)$ & $<1.4(-08)$ & $3.1(16)$ & $1.1(17)$ & $<4.4(-09)$ \\
\hline $\mathrm{SiC}_{2}(4,04-3,03)$ & $1.1(16)$ & $2.8(16)$ & $2.3(-07)$ & $8.6(15)$ & $2.2(16)$ & - & $3.1(16)$ & $7.8(16)$ & - & $3.1(16)$ & $7.8(16)$ & - \\
\hline $\mathrm{SiC}_{2}(4,22-3,21)$ & $1.1(16)$ & $2.8(16)$ & $<2.7(-07)$ & $8.6(15)$ & $2.2(16)$ & - & $3.1(16)$ & $7.8(16)$ & - & $3.1(16)$ & $7.8(16)$ & - \\
\hline $\mathrm{SiC}_{2}(5,05-4,04)$ & $1.1(16)$ & $2.8(16)$ & $<3.2(-07)$ & $8.6(15)$ & $2.2(16)$ & $3.1(-06)$ & $3.1(16)$ & $7.8(16)$ & $<7.5(-08)$ & $3.1(16)$ & $7.8(16)$ & $<5.3(-08)$ \\
\hline $\mathrm{C}_{2} \mathrm{H}(1-0)$ & $1.1(16)$ & $3.2(16)$ & $<4.3(-07)$ & $1.4(16)$ & $4.1(16)$ & $6.9(-06)$ & $2.8(16)$ & $6.7(16)$ & $5.7(-06)$ & $2.7(16)$ & $6.7(16)$ & $<1.0(-07)$ \\
\hline $\mathrm{C}_{3} \mathrm{H}(9 / 2-7 / 2)$ & $1.1(16)$ & $4.0(16)$ & $<3.1(-08)$ & $8.6(15)$ & $3.0(16)$ & $<7.4(-08)$ & $3.1(16)$ & $1.1(17)$ & $<1.4(-08)$ & $3.1(16)$ & $1.1(17)$ & $<1.2(-08)$ \\
\hline $\mathrm{C}_{3} \mathrm{~N}(9-8)$ & $1.1(16)$ & $4.0(16)$ & $<1.6(-07)$ & $8.6(15)$ & $3.0(16)$ & - & $3.1(16)$ & $1.1(17)$ & - & $3.1(16)$ & $1.1(17)$ & - \\
\hline $\mathrm{C}_{3} \mathrm{~N}(11-10)$ & $1.1(16)$ & $4.0(16)$ & $7.3(-07)$ & $8.6(15)$ & $3.0(16)$ & $2.6(-06)$ & $3.1(16)$ & $1.1(17)$ & $5.5(-07)$ & $3.1(16)$ & $1.1(17)$ & $1.1(-07)$ \\
\hline $\mathrm{C}_{4} \mathrm{H}(9-8)$ & $1.1(16)$ & $4.0(16)$ & - & $8.6(15)$ & $3.0(16)$ & - & $3.1(16)$ & $1.1(17)$ & - & $3.1(16)$ & $1.1(17)$ & - \\
\hline $\mathrm{C}_{4} \mathrm{H}(10-9)$ & $1.1(16)$ & $4.0(16)$ & $<8.8(-07)$ & $8.6(15)$ & $3.0(16)$ & $<1.7(-06)$ & $3.1(16)$ & $1.1(17)$ & - & $3.1(16)$ & $1.1(17)$ & - \\
\hline $\mathrm{C}_{3} \mathrm{H}_{2}(2,12-1,01)$ & $1.1(16)$ & $4.0(16)$ & - & $8.6(15)$ & $3.0(16)$ & - & $3.1(16)$ & $1.1(17)$ & - & $3.1(16)$ & $1.1(17)$ & - \\
\hline $\mathrm{HC}_{3} \mathrm{~N}(10-9)$ & $5.6(15)$ & $2.8(16)$ & $1.7(-06)$ & $4.3(15)$ & $2.2(16)$ & $2.4(-06)$ & $1.6(16)$ & $7.8(16)$ & $5.0(-07)$ & $1.6(16)$ & $7.8(16)$ & $1.5(-06)$ \\
\hline $\mathrm{HCC}^{13} \mathrm{CN}(10-9)$ & $5.6(15)$ & $2.8(16)$ & $1.5(-07)$ & $4.3(15)$ & $2.2(16)$ & $<4.9(-08)$ & $1.6(16)$ & $7.8(16)$ & $<1.5(-08)$ & $1.6(16)$ & $7.8(16)$ & $3.1(-08)$ \\
\hline $\mathrm{HC}^{13} \mathrm{CCN}(10-9)$ & $5.6(15)$ & $2.8(16)$ & $1.5(-07)$ & $4.3(15)$ & $2.2(16)$ & $<4.9(-08)$ & $1.6(16)$ & $7.8(16)$ & $<1.5(-08)$ & $1.6(16)$ & $7.8(16)$ & $3.1(-08)$ \\
\hline $\mathrm{HC}_{3} \mathrm{~N}(11-10)$ & $5.6(15)$ & $2.8(16)$ & - & $4.3(15)$ & $2.2(16)$ & - & $1.6(16)$ & $7.8(16)$ & - & $1.6(16)$ & $7.8(16)$ & - \\
\hline $\mathrm{HC}_{3} \mathrm{~N}(12-11)$ & $5.6(15)$ & $2.8(16)$ & $2.4(-06)$ & $4.3(15)$ & $2.2(16)$ & - & $1.6(16)$ & $7.8(16)$ & - & $1.6(16)$ & $7.8(16)$ & - \\
\hline $\mathrm{HCC}^{13} \mathrm{CN}(12-11)$ & $5.6(15)$ & $2.8(16)$ & $1.3(-07)$ & $4.3(15)$ & $2.2(16)$ & $2.7(-07)$ & $1.6(16)$ & $7.8(16)$ & $<2.9(-08)$ & $1.6(16)$ & $7.8(16)$ & $<1.1(-08)$ \\
\hline $\mathrm{HC}^{13} \mathrm{CCN}(12-11)$ & $5.6(15)$ & $2.8(16)$ & $1.3(-07)$ & $4.3(15)$ & $2.2(16)$ & $2.7(-07)$ & $1.6(16)$ & $7.8(16)$ & $<2.9(-08)$ & $1.6(16)$ & $7.8(16)$ & $<1.1(-08)$ \\
\hline $\mathrm{CH}_{3} \mathrm{CN}(6(1)-5(1))$ & $5.6(15)$ & $2.8(16)$ & $<2.4(-07)$ & $4.3(15)$ & $2.2(16)$ & $<1.3(-07)$ & $1.6(16)$ & $7.8(16)$ & $<1.2(-07)$ & $1.6(16)$ & $7.8(16)$ & $<6.4(-08)$ \\
\hline $\mathrm{CH}_{3} \mathrm{CN}(12(0)-11(0))$ & $5.6(15)$ & $2.8(16)$ & $<4.6(-06)$ & $4.3(15)$ & $2.2(16)$ & - & $1.6(16)$ & $7.8(16)$ & - & $1.6(16)$ & $7.8(16)$ & - \\
\hline $\mathrm{HC}_{5} \mathrm{~N}(32-31)$ & $5.6(15)$ & $2.8(16)$ & - & $4.3(15)$ & $2.2(16)$ & - & $1.6(16)$ & $7.8(16)$ & - & $1.6(16)$ & $7.8(16)$ & - \\
\hline $\mathrm{HC}_{5} \mathrm{~N}(34-33)$ & $5.6(15)$ & $2.8(16)$ & $9.2(-06)$ & $4.3(15)$ & $2.2(16)$ & $<1.3(-05)$ & $1.6(16)$ & $7.8(16)$ & $<4.1(-06)$ & $1.6(16)$ & $7.8(16)$ & $<4.5(-06)$ \\
\hline $\mathrm{HC}_{5} \mathrm{~N}(35-34)$ & $5.6(15)$ & $2.8(16)$ & $<8.8(-06)$ & $4.3(15)$ & $2.2(16)$ & - & $1.6(16)$ & $7.8(16)$ & - & $1.6(16)$ & $7.8(16)$ & - \\
\hline
\end{tabular}

${ }^{a}$ In this table $x(y)$ represents $x \times 10^{y}$.

${ }^{b}$ Bold face indicates an abundance calculated by scaling the abundance of the ${ }^{13} \mathrm{C}$ isotope of the same species by the modelled

${ }^{12} \mathrm{CO} /{ }^{13} \mathrm{CO}$-ratio (see Sect. 5.5).

of AGB-stars induces pulsation-driven shocks that propagate outwards and suppress TE from the point of shock formation. Non-equilibrium chemical modelling has been performed by Willacy \& Cherchneff $(1998$, for IRC +10216$)$, and shows that shocks can strongly alter the chemical abundances in the inner regions of the CSE from their TE values. In particular, $\mathrm{SiO}$ is strongly enhanced whereas HCN and CS are destroyed. Other species, e.g., $\mathrm{CO}$ and $\mathrm{SiS}$ are relatively unaffected.

In Table 10 the abundances obtained by Willacy \& Cherchneff (1998) for TE and non-equilibrium chemical modelling (shock strength of $11.7 \mathrm{~km} \mathrm{~s}^{-1}$ ) are compared to the values obtained in the analysis. Given the uncertanties, about a factor of five, the abundances obtained from the observations clearly favour a scenario in which a shock has passed through the inner $\left(\$ 5 R_{\star}\right)$ parts of the wind. The $\mathrm{SiO}$ and $\mathrm{SiS}$ abundances which are derived are lower limits since they could be significantly depleted in the outer envelope due to freeze-out onto dust grains. The average abundances derived for $\mathrm{HCN}$, $\mathrm{CS}$, and $\mathrm{SiO}$ in the sample are $1 \times 10^{-5}, 1.5 \times 10^{-6}, 7 \times 10^{-7}$, respectively. The $\mathrm{SiO}$ abundance shows the largest spread among the sources reflecting its sensitivity to the shock strength and possible variation in the $\mathrm{C} / \mathrm{O}$-ratio among the sample sources. CS might, however, not be particularly well suited as a probe of shocked chemistry. Olofsson et al. (1993a) found, when modelling a large sample of optically bright carbon stars, that in their photospheric LTE models the CS abundance varied considerably with adopted stellar temperature.

\subsubsection{Photochemistry in the outer envelope}

Many photochemical models have been developed for the outer envelope of IRC +10216 (e.g. Millar \& Herbst 1994; 
Table 8. Molecular isotope abundance ratios.

\begin{tabular}{lcccccc}
\hline \hline & IRC+10216 (S) & IRC+10216 (O) & IRAS 15194 & IRAS 07454 & CIT 6 & IRC+40540 \\
\hline $\mathrm{HC}_{3} \mathrm{~N} / \mathrm{HC}^{13} \mathrm{CCN}(10-9)$ & 32.6 & - & 4.9 & 11.1 & - & 17.9 \\
$\mathrm{HC}_{3} \mathrm{~N} / \mathrm{HC}^{13} \mathrm{CCN}(12-11)$ & 22.1 & - & 7.9 & 17.8 & - & - \\
$\mathrm{CS} /{ }^{13} \mathrm{CS}(2-1)$ & 22.7 & - & 4.6 & - & - & - \\
$\mathrm{CN} /{ }^{13} \mathrm{CN}(1-0)$ & 25.1 & 46.6 & 6.7 & 11.0 & 12.4 & - \\
Average & 25.6 & 46.6 & 6.0 & 13.3 & 12.4 & 17.9 \\
Modelling of CO (Table 4) & 45.0 & 45.0 & 6.0 & 14.0 & 35.0 & 50.0 \\
\hline
\end{tabular}

Table 9. Abundance ratios, compared to IRC +10216 observed with the SEST.

\begin{tabular}{|c|c|c|c|c|c|c|c|c|}
\hline \multirow[b]{2}{*}{ Molecule } & \multicolumn{2}{|c|}{ IRC +10216 } & \multirow[t]{2}{*}{ IRAS 15194} & \multirow[t]{2}{*}{ IRAS 15082} & \multirow[t]{2}{*}{ IRAS 07454} & \multirow[t]{2}{*}{ CIT 6} & \multirow[t]{2}{*}{ AFGL 3068} & \multirow[t]{2}{*}{ IRC +40540} \\
\hline & SEST & OSO & & & & & & \\
\hline $\mathrm{CN}(1-0)$ & 1.0 & 1.1 & 1.1 & 1.7 & 2.8 & 3.8 & 0.2 & 0.5 \\
\hline${ }^{13} \mathrm{CN}(1-0)$ & 1.0 & 0.6 & 4.0 & - & 6.3 & 7.6 & - & - \\
\hline $\mathrm{CN}(2-1)$ & 1.0 & - & 0.4 & 0.4 & 1.7 & - & - & - \\
\hline $\mathrm{CS}(2-1)$ & 1.0 & 0.9 & 4.6 & 4.3 & 3.2 & 5.1 & 0.7 & 1.1 \\
\hline${ }^{13} \mathrm{CS}(2-1)$ & 1.0 & - & 22.7 & - & - & - & - & - \\
\hline $\operatorname{CS}(5-4)$ & 1.0 & - & 1.4 & 0.8 & 1.4 & - & - & - \\
\hline $\mathrm{SiO}(2-1)$ & 1.0 & 0.7 & 12.8 & 5.4 & 3.3 & 7.8 & - & 0.4 \\
\hline $\operatorname{SiS}(5-4)$ & 1.0 & 0.7 & 4.0 & 2.7 & 3.9 & 0.8 & 0.3 & 1.1 \\
\hline $\operatorname{SiS}(6-5)$ & 1.0 & 1.0 & 4.7 & 2.2 & 3.8 & 5.7 & 1.1 & 0.5 \\
\hline $\mathrm{HCN}$ & 1.0 & 0.8 & 0.9 & 0.7 & 0.6 & 0.8 & 0.5 & 0.5 \\
\hline $\mathrm{H}^{13} \mathrm{CN}(1-0)$ & 1.0 & 0.8 & 6.4 & 0.9 & 1.8 & 1.0 & 0.7 & 0.4 \\
\hline $\mathrm{HNC}(1-0)$ & 1.0 & 0.5 & 4.6 & 2.2 & 1.4 & 3.2 & 0.4 & 0.3 \\
\hline $\mathrm{SiC}_{2}(4,04-3,03)$ & 1.0 & - & - & 2.0 & 1.5 & - & - & - \\
\hline $\mathrm{SiC}_{2}(5,05-4,04)$ & 1.0 & 1.0 & 6.9 & 2.8 & - & 17.7 & - & - \\
\hline $\mathrm{C}_{2} \mathrm{H}(1-0)$ & 1.0 & 0.9 & 5.6 & 3.0 & - & 2.5 & 2.0 & - \\
\hline $\mathrm{C}_{3} \mathrm{H}(9 / 2-7 / 2)$ & 1.0 & 1.1 & 1.7 & - & - & - & - & - \\
\hline $\mathrm{C}_{3} \mathrm{~N}(11-10)$ & 1.0 & 1.3 & 1.5 & 1.6 & 1.2 & 4.4 & 0.9 & 0.2 \\
\hline $\mathrm{C}_{4} \mathrm{H}(10-9)$ & 1.0 & 1.2 & 10.4 & 2.6 & - & - & - & - \\
\hline $\mathrm{C}_{3} \mathrm{H}_{2}(2,12-1,01)$ & 1.0 & - & 18.6 & - & - & - & - & - \\
\hline $\mathrm{HC}_{3} \mathrm{~N}(10-9)$ & 1.0 & 0.6 & 2.4 & 3.0 & 2.2 & 3.1 & 0.6 & 0.7 \\
\hline $\mathrm{H}^{(13)} \mathrm{C}_{3} \mathrm{~N}(10-9)^{a}$ & 1.0 & - & 16.3 & - & 6.4 & - & - & 1.3 \\
\hline $\mathrm{HC}_{3} \mathrm{~N}(12-11)$ & 1.0 & - & 2.5 & 2.3 & 2.6 & - & - & - \\
\hline $\mathrm{H}^{(13)} \mathrm{C}_{3} \mathrm{~N}(12-11)^{a}$ & 1.0 & 0.4 & 6.9 & - & 3.3 & 6.6 & - & - \\
\hline $\mathrm{HC}_{5} \mathrm{~N}(34-33)$ & 1.0 & 一 & - & - & 2.2 & 一 & - & - \\
\hline
\end{tabular}

Bold face signifies a factor of more than 5 .

${ }^{a}$ signifies blend of $\mathrm{HCC}^{13} \mathrm{CN}$ and $\mathrm{HC}^{13} \mathrm{CCN}$.

Doty \& Leung 1998; Millar et al. 2000). The physical conditions in the outer parts of the wind $\left(\gtrsim 100 R_{\star}\right)$ allow for the penetration of ambient ultraviolet radiation that induces a photochemistry. In Table 11 the derived column densities for IRC +10216 for a number of species produced in the envelope are compared to those from the photochemical model of Millar et al. (2000). In general the abundances agree well, given the uncertainties.

It is remarkable that the abundances generally show relatively little variation within the sample. Most apparent is the over-abundance of Si-bearing molecules in CIT 6 (Sect. 6.3). Some of the abundances of IRAS 15194-5115 also stand out and will be separately discussed in Sect. 6.2. There are no apparent trends with the stellar or circumstellar parameters. This would suggest that the physical structure in these sources indeed is much the same and that the initial atomic abundances are similar. When comparing the present sample of carbon stars to the sample of Olofsson et al. (1993a), which on the whole tend to have a low mass-loss rate $\left(\sim 10^{-7} M_{\odot} \mathrm{yr}^{-1}\right)$, the agreement in derived abundances for the star in common, CIT 6, is very good. There is less than a factor 3 difference in the calculated abundances of $\mathrm{HCN}, \mathrm{CN}$ and CS. However, in general, the sample of low mass-loss rate stars has calculated abundances which are systematically an order of magnitude greater than those calculated here. It must be noted that Olofsson et al. (1993a) use an excitation temperature of $20 \mathrm{~K}$, twice that used in this analysis. Moreover, Schöier \& Olofsson (2001) show that the mass-loss rates in the low mass-loss rate objects in Olofsson et al. (1993a) are underestimated by about a factor of 5 on average. This would explain the apparent discrepancy in calculated abundances between the high mass-loss rate objects here and the lower mass-loss rate objects in Olofsson et al. (1993a). Hence there seems not to be a marked difference in the molecular composition of high and low mass-loss carbon stars. 
Table 10. Fractional abundances for IRC +10216 for species of photospheric origin.

\begin{tabular}{llcc}
\hline \hline Species & Observed $^{a}$ & \multicolumn{2}{c}{ Model $^{b}$} \\
\cline { 3 - 4 } & & TE & Shock \\
\hline $\mathrm{CO}$ & $1.0(-3)^{c}$ & $9.8(-4)$ & $1.0(-3)$ \\
$\mathrm{HCN}$ & $1.3(-5)$ & $5.1(-5)$ & $3.1(-6)$ \\
$\mathrm{CS}$ & $9.9(-7)$ & $1.3(-5)$ & $7.1(-7)$ \\
$\mathrm{SiS}$ & $9.5(-7)$ & $1.0(-5)$ & $2.5(-5)$ \\
$\mathrm{SiO}$ & $1.1(-7)$ & $1.9(-8)$ & $2.7(-7)$ \\
\hline
\end{tabular}

${ }^{a}$ From Table 7.

${ }^{b}$ From chemical modelling by Willacy \& Cherchneff (1998).

${ }^{c}$ Assumed value that determines molecular hydrogen densities.

The large spread in the abundance of isotopomers containing ${ }^{13} \mathrm{C}$ follows from the varying ${ }^{12} \mathrm{C} /{ }^{13} \mathrm{C}$-ratio among the sample sources. The ${ }^{12} \mathrm{C} /{ }^{13} \mathrm{C}$-ratio is related to the nucleosynthesis rather than the chemistry and reflects the evolutionary status of these stars. However, chemical fractionation may affect this ratio in certain molecules, in particular $\mathrm{CO}$.

$\mathrm{CN} / \mathrm{HCN}$ and $\mathrm{HNC} / \mathrm{HCN}$ ratios can also be used to estimate the evolutionary status of carbon stars. $\mathrm{CN}$ is produced via the photodissociation of $\mathrm{HCN}$ by ultraviolet radiation, and as stellar radiation increases with evolution from AGB star to PPN to PN, so the $\mathrm{CN} / \mathrm{HCN}$ ratio will increase (e.g., Bachiller et al. 1997a; Cox et al. 1992). HNC, formed from the dissociative recombination of $\mathrm{HCNH}^{+}$, behaves in a similar way. In this sample there is a rather large spread in the $\mathrm{CN} / \mathrm{HCN}$ ratio, from 0.07-0.68, and a value of 1.82 for CIT 6 (see Sect. 6.3). This large spread is in contrast to Olofsson et al. (1993a), for example, who found a very narrow range $(\mathrm{CN} / \mathrm{HCN} \sim 0.65-0.70)$ in low mass-loss stars. However, there is excellent agreement with the results of Lindqvist et al. (2000). For the carbon stars IRC +10216 , CIT 6 and IRC +40540 they derive ratios of 0.16 , 1.5 and 0.17 , respectively, in comparison with the $0.22,1.82$ and 0.15 derived here. $\mathrm{A} \mathrm{CN} / \mathrm{HCN}$ ratio of $\sim 0.5$ is typical in C-rich AGB stars (Bachiller et al. 1997b), increasing to $\sim 5$ in PPNe. In fact, a ratio of $0.6-0.7$ is predicted by the photodissociation model, with only a weak dependence on mass-loss rate (see Fig. 8 of Lindqvist et al. 2000). The HNC/HCN ratio seems to be split into two ranges in the present sample of carbon stars. IRC +10216, AFGL 3068 and IRC +40540 have $\mathrm{HNC} / \mathrm{HCN}$ ratios of $\leq 0.005$, whilst the remaining stars have ratios of $0.01-0.03$. The value derived for IRC +10216 is in agreement with that quoted in Cox et al. (1992). This seems to indicate that the sample stars are not well evolved, since a $\mathrm{HNC} / \mathrm{HCN}$ ratio of $\sim 1$ is expected in PPNe (e.g., Cox et al. 1992). Having said this, the $\mathrm{HNC} / \mathrm{HCN}$ ratio does rapidly become of the order 1, as can be seen in models of PPN chemistry (Woods et al. 2003).

Generally, it seems that to use the term "carbon chemistry" to refer to a paradigm of chemistry in C-rich evolved stars is reasonable. Of the sample stars here, given the variety of molecular species, there is very little difference in molecular abundances, save for two slightly curious sources, as detailed in the following subsections.
Table 11. Radial column densities $\left(\mathrm{cm}^{-2}\right)$ for species of circumstellar origin towards IRC +10216 .

\begin{tabular}{lccc}
\hline \hline Species & Observed $^{a}$ & MHB $^{b}$ & Obs./MHB \\
\hline $\mathrm{CN}$ & $8.3(14)$ & $1.0(15)$ & 0.8 \\
$\mathrm{HNC}$ & $2.0(13)$ & $8.4(13)$ & 0.2 \\
$\mathrm{C}_{2} \mathrm{H}$ & $8.9(14)$ & $5.7(15)$ & 0.2 \\
$\mathrm{C}_{3} \mathrm{H}$ & $2.1(13)$ & $1.4(14)$ & 0.2 \\
$\mathrm{C}_{3} \mathrm{~N}$ & $2.0(14)$ & $3.2(14)$ & 0.6 \\
$\mathrm{C}_{4} \mathrm{H}$ & $9.3(14)$ & $1.0(15)$ & 0.9 \\
$\mathrm{C}_{3} \mathrm{H}_{2}$ & $1.2(13)$ & $2.1(13)$ & 0.6 \\
$\mathrm{HC}_{3} \mathrm{~N}$ & $9.1(14)$ & $1.8(15)$ & 0.5 \\
$\mathrm{CH}_{3} \mathrm{CN}$ & $9.9(12)$ & $3.4(12)$ & 2.9 \\
$\mathrm{HC}_{5} \mathrm{~N}$ & $5.8(15)$ & $7.1(14)$ & 8.2 \\
\hline
\end{tabular}

${ }^{a}$ Calculated from Tables 4 and 7.

${ }^{b}$ From chemical modelling by Millar et al. (2000).

\subsection{IRAS $15194-5115$}

Of the derived abundances those obtained for IRAS 151945115 stand out the most. In particular, the $\mathrm{SiO}$ and $\mathrm{C}_{4} \mathrm{H}$ abundances, in addition to the isotopomers containing ${ }^{13} \mathrm{C}$, appear significantly enhanced towards this source. $\mathrm{C}_{3} \mathrm{H}_{2}$ also appears to be greatly enhanced in this source, but, however, this molecule is only observed in one other star, IRC +10216 . The ${ }^{12} \mathrm{CO} /{ }^{13} \mathrm{CO}$-ratio of 6 derived for IRAS $15194-5115$ is significantly lower than that of the others and that which is commonly derived for carbon stars. This value is certain, with the modelling of the $\mathrm{CO}$ emission being supported by intensity ratios for another four species, which agree to $\pm 30 \%$ (Table 8). The evolutionary status of this star is undetermined. Ryde et al. (1999) speculated that IRAS 15194-5115 might be a massive (5-8 $\left.M_{\odot}\right)$ star in the last stages of evolution where its low ${ }^{12} \mathrm{C} /{ }^{13} \mathrm{C}$-ratio is the result of hot bottom burning (HBB). The increase of ${ }^{14} \mathrm{~N}$ from the $\mathrm{CNO}$ cycle is a signature of $\mathrm{HBB}$ (Marigo 2001; Ventura et al. 2002), but given the uncertainty in the data presented here, this suggestion cannot be confirmed.

\section{3. $C I T 6$}

CIT 6 is another object outstanding in the sample. It has a $\mathrm{CN} / \mathrm{HCN}$ ratio of $\sim 1.8$, which suggests an advanced evolutionary status, but a low $\mathrm{HNC} / \mathrm{HCN}$ ratio $(\sim 0.02)$, which suggests the contrary. Certainly the idea that CIT 6 is well on its way to becoming a PPN has been put forward before (e.g., Trammell et al. 1994; Monnier et al. 2000; Začs et al. 2001).

The modelled ${ }^{12} \mathrm{CO} /{ }^{13} \mathrm{CO}$ ratio in this source agrees well with that carried out previously (Groenewegen et al. 1996; Schöier \& Olofsson 2000). This ratio, however, does not agree with ${ }^{12} \mathrm{C} /{ }^{13} \mathrm{C}$ ratios derived from observations of other molecules and their ${ }^{13} \mathrm{C}$ isotopes, both in this paper (Table 8) and elsewhere (Kahane et al. 1992; Groenewegen et al. 1996).

A further point worth note is the comparative overabundance of Si-bearing species which possibly indicates a less efficient freeze-out onto dust grains in this particular source. 


\section{Conclusions}

The seven high mass-loss rate carbon stars presented here exhibit rich spectra at millimetre wavelengths with many molecular species readily detected. A total of 47 emission lines from 24 molecular species were detected for the sample stars. The mass-loss rate and physical structure of the circumstellar envelope, such as the density and temperature profiles, was carefully estimated based upon a detailed radiative transfer analysis of CO. The determination of the mass-loss rate enables abundances for the remaining molecular species to be calculated. The derived abundances typically agree within a factor of five indicating that circumstellar envelopes around carbon stars have similar molecular compositions.

The most striking difference between the abundances are reflecting the spread in the ${ }^{12} \mathrm{C} /{ }^{13} \mathrm{C}$-ratio of about an order of magnitude between the sample stars. Also, the high abundance of $\mathrm{SiO}$ in the envelopes indicates that a shock has passed through the gas in the inner parts of the envelope. This is further corroborated by the relatively low amounts of CS and possibly HCN.

The abundances of species that are produced in the outer parts of the wind can be reasonably well explained by current photochemical models.

Acknowledgements. We are grateful to F. Kerschbaum for help with estimating some of the input parameters to the $\mathrm{CO}$ modelling. FLS is supported by the Netherlands Organization for Scientific Research (NWO) grant 614.041.004. The Swedish-ESO Submillimetre Telescope, SEST, is operated jointly by ESO and the Swedish National Facility for Radioastronomy, Onsala Space Observatory at Chalmers University of Technology. The OSO $20 \mathrm{~m}$ telescope is operated by the Swedish National Facility for Radioastronomy. This article made use of data obtained through the JCMT archive as Guest User at the Canadian Astronomy Data Center, which is operated by the Dominion Astrophysical Observatory for the National Research Council of Canada's Herzberg Institute of Astrophysics.

\section{References}

Abia, C., Busso, M., Gallino, R., et al. 2001, ApJ, 559, 1117

Bachiller, R., Forveille, T., Huggins, P. J., \& Cox, P. 1997a, A\&A, 324,1123

Bachiller, R., Fuente, A., Bujarrabal, V., et al. 1997b, A\&A, 319, 235

Bernes, C. 1979, A\&A, 73, 67

Bieging, J. H., \& Rieu, N. 1988, ApJ, 329, L107

Bieging, J. H., \& Tafalla, M. 1993, AJ, 105, 576

Booth, R. S., Delgado, G., Hagstrom, M., et al. 1989, A\&A, 216, 315

Boucher, D., Burie, J., Bauer, A., Dubrulle, A., \& Demaison, J. 1980, J. Phys. Chem. Ref. Data, 9, 659

Bujarrabal, V., Fuente, A., \& Omont, A. 1994, A\&A, 285, 247

Cernicharo, J., Guélin, M., \& Kahane, C. 2000, A\&AS, 142, 181

Chandra, S., Maheshwari, V. U., \& Sharma, A. K. 1996, A\&AS, 117, 557

Chandra, S., \& Rashmi. 1998, A\&AS, 131, 137

Cox, P., Omont, A., Huggins, P. J., Bachiller, R., \& Forveille, T. 1992, A\&A, 266, 420
Crosas, M., \& Menten, K. M. 1997, ApJ, 483, 913

Dayal, A., \& Bieging, J. H. 1993, ApJ, 407, L37

Dayal, A., \& Bieging, J. H. 1995, ApJ, 439, 996

Doty, S. D., \& Leung, C. M. 1998, ApJ, 502, 898

Flower, D. R. 2001, J. Phys. B: Mol. Opt. Phys., 34, 1

Gensheimer, P. D., Likkel, L., \& Snyder, L. E. 1995, ApJ, 439, 445

Glassgold, A. E. 1996, ARA\&A, 34, 241

Groenewegen, M. A. T. 1994, A\&A, 290, 531

Groenewegen, M. A. T., Baas, F., de Jong, T., \& Loup, C. 1996, A\&A, 306,241

Groenewegen, M. A. T., \& Whitelock, P. A. 1996, MNRAS, 281, 1347

Guelin, M., Lucas, R., \& Cernicharo, J. 1993, A\&A, 280, L19

Guelin, M., Lucas, R., \& Neri, R. 1996, in CO: Twenty-Five Years of Millimeter-Wave Spectroscopy, IAU Symp., 170, 359

Huggins, P. J., \& Glassgold, A. E. 1982, ApJ, 252, 201

Iben, I., \& Renzini, A. 1983, ARA\&A, 21, 271

Jura, M., \& Morris, M. 1981, ApJ, 251, 181

Kahane, C., Cernicharo, J., Gomez-Gonzalez, J., \& Guelin, M. 1992, A\&A, 256, 235

Kahane, C., Gomez-Gonzalez, J., Cernicharo, J., \& Guelin, M. 1988, A\&A, 190, 167

Kastner, J. H. 1992, ApJ, 401, 337

Kerschbaum, F. 1999, A\&A, 351, 627

Lindqvist, M., Schöier, F. L., Lucas, R., \& Olofsson, H. 2000, A\&A, 361, 1036

Lucas, R., \& Guélin, M. 1999, in Asymptotic Giant Branch Stars, IAU Symp., 191, 305

Lucas, R., Guelin, M., Kahane, C., Audinos, P., \& Cernicharo, J. 1995, Ap\&SS, 224, 293

Mamon, G. A., Glassgold, A. E., \& Huggins, P. J. 1988, ApJ, 328, 797

Marigo, P. 2001, A\&A, 370, 194

Men'shchikov, A. B., Balega, Y., Blöcker, T., Osterbart, R., \& Weigelt, G. 2001, A\&A, 368, 497

Millar, T. J., Flores, J. R., \& Markwick, A. J. 2001, MNRAS, 327, 1173

Millar, T. J., \& Herbst, E. 1994, A\&A, 288, 561

Millar, T. J., Herbst, E., \& Bettens, R. P. A. 2000, MNRAS, 316, 195

Monnier, J. D., Tuthill, P. G., \& Danchi, W. C. 2000, ApJ, 545, 957

Nejad, L. A. M., \& Millar, T. J. 1988, MNRAS, 230, 79

Neri, R., Kahane, C., Lucas, R., Bujarrabal, V., \& Loup, C. 1998, A\&AS, 130, 1

Nyman, L.-A., Booth, R. S., Carlstrom, U., et al. 1992, A\&AS, 93, 121

Nyman, L.-A., Olofsson, H., Johansson, L. E. B., et al. 1993, A\&A, 269, 377

Olofsson, H., Eriksson, K., \& Gustafsson, B. 1990, A\&A, 230, 405

Olofsson, H., Eriksson, K., Gustafsson, B., \& Carlstroem, U. 1993a, ApJS, 87, 305

Olofsson, H., Eriksson, K., Gustafsson, B., \& Carlstrom, U. 1993b, ApJS, 87, 267

Olofsson, H., González Delgado, D., Kerschbaum, F., \& Schöier, F. L. 2002, A\&A, 391, 1053

Olofsson, H., Lindqvist, M., Nyman, L.-A., \& Winnberg, A. 1998, A\&A, 329, 1059

Ryde, N., Schöier, F. L., \& Olofsson, H. 1999, A\&A, 345, 841

Sahai, R. 1990, ApJ, 362, 652

Schöier, F. L. 2000, Ph.D. Thesis, Stockholm Observatory, SE-133 36 Saltsjöbaden, Sweden

Schöier, F. L., \& Olofsson, H. 2000, A\&A, 359, 586

Schöier, F. L., \& Olofsson, H. 2001, A\&A, 368, 969

Schöier, F. L., Ryde, N., \& Olofsson, H. 2002, A\&A, 391, 577

Schinke, R., Engel, V., Buck, U., Meyer, H., \& Diercksen, G. H. F. 1985, ApJ, 299, 939 
Suh, K. 2000, MNRAS, 315, 740

Townes, C. H., \& Schawlow, A. L. 1975, Microwave Spectroscopy (New York: Dover)

Trammell, S. R., Dinerstein, H. L., \& Goodrich, R. W. 1994, AJ, 108, 984

Truong-Bach, Nguyen-Q-Rieu, Omont, A., Olofsson, H., \& Johansson, L. E. B. 1987, A\&A, 176, 285

van Dishoeck, E. F. 1988, in Rate Coefficients in Astrochemistry, ed. T. J. Millar \& D. A. Williams (Kluwer: Academic Publishers), ASSL, 146, 49 van Zadelhoff, G.-J., Dullemond, C. P., van der Tak, F. F. S., et al. 2002, A\&A, 395, 373

Vassiliadis, E., \& Wood, P. R. 1993, ApJ, 413, 641

Ventura, P., D’Antona, F., \& Mazzitelli, I. 2002, A\&A, 393, 215

Vrtilek, J. M., Gottlieb, C. A., \& Thaddeus, P. 1987, ApJ, 314, 716

Willacy, K., \& Cherchneff, I. 1998, A\&A, 330, 676

Woods, P. M., Millar, T. J., Herbst, E., \& Zijlstra, A. A. 2003, A\&A, in press

Začs, L., Alksnis, A., \& Spëlmanis, R. 2001, in Post-AGB Objects as a Phase of Stellar Evolution, 289 